Y.-F. S. PÉTERMANN

\title{
On an estimate of Walfisz and Saltykov for an error term related to the Euler function
}

Journal de Théorie des Nombres de Bordeaux, tome 10, $\mathrm{n}^{\circ} 1$ (1998), p. 203-236

<http://www.numdam.org/item?id=JTNB_1998_10_1_203_0>

(C) Université Bordeaux 1, 1998, tous droits réservés.

L'accès aux archives de la revue «Journal de Théorie des Nombres de Bordeaux » (http://jtnb.cedram.org/) implique l'accord avec les conditions générales d'utilisation (http://www.numdam.org/conditions). Toute utilisation commerciale ou impression systématique est constitutive d'une infraction pénale. Toute copie ou impression de ce fichier doit contenir la présente mention de copyright.

\section{Numdam}

Article numérisé dans le cadre du programme

Numérisation de documents anciens mathématiques

http://www.numdam.org/ 


\title{
On an estimate of Walfisz and Saltykov for an error term related to the Euler function
}

\author{
par Y.-F. S. PÉTERMANN
}

RÉSUMÉ. On étend la technique qui a permis à $\mathrm{A}$. Walfisz d'établir (en 1962) l'estimation $H(x) \ll(\log x)^{2 / 3}(\log \log x)^{4 / 3}$ pour le terme d'erreur lié à la fonction d'Euler $H(x)=\sum_{n \leq x} \frac{\phi(n)}{n}-\frac{6}{\pi^{2}} x$, tout en incorporant à l'argument des simplifications rendues possibles par des travaux de A.I. Saltykov et de A.A. Karatsuba.

On remarque en passant que la preuve proposée en 1960 par Saltykov de $H(x) \ll(\log x)^{2 / 3}(\log \log x)^{1+\epsilon}$ contient une faute, qui une fois corrigée ne livre "que" le résultat de Walfisz.

Les généralisations obtenues s'appliquent aux termes d'erreurs liés à diverses fonctions arithmétiques classiques, et moins classiques, comme par exemple à $(\phi(n) / n)^{r},(\sigma(n) / n)^{r}$ et $(\sigma(n) / \phi(n))^{r}$ pour chaque valeur réelle de $r$, ou encore à $\sigma^{(r)}(n)$, la somme des diviseurs exponentiels $d$ de $n$ tels que $p^{\alpha} \not \| d$ si $p^{2 \alpha} \| n$ et $\alpha>1$.

AbSTRACT. The technique developed by A. Walfisz in order to prove (in 1962) the estimate $H(x) \ll(\log x)^{2 / 3}(\log \log x)^{4 / 3}$ for the error term $H(x)=\sum_{n \leq x} \frac{\phi(n)}{n}-\frac{6}{\pi^{2}} x$ related to the Euler function is extended. Moreover, the argument is simplified by exploiting works of A.I. Saltykov and of A.A. Karatsuba.

It is noted in passing that the proof proposed by Saltykov in 1960 of $H(x) \ll(\log x)^{2 / 3}(\log \log x)^{1+\epsilon}$ is erroneous and once corrected "only" yields Walfisz' result.

The generalizations obtained can be applied to error terms related to various classical - and less classical - arithmetical functions, as for instance to $(\phi(n) / n)^{r},(\sigma(n) / n)^{r}$ and $(\sigma(n) / \phi(n))^{r}$ for every real value of $r$, and also to $\sigma^{(r)}(n)$, the sum of the exponential divisors $d$ of $n$ with $p^{\alpha} \not \mid d$ if $p^{2 \alpha} \| n$ and $\alpha>1$. 


\section{INTRODUCTION}

Let $\phi$ denote as usual the Euler totient function, and consider the associated error term

$$
H(x)=\sum_{n \leq x} \frac{\phi(n)}{n}-\frac{6}{\pi^{2}} x .
$$

It was already known to Dirichlet in 1849 that $H(x)=o(x)$. A proof of the more precise estimate

$$
H(x)=O\left((\log x)^{2 / 3}(\log \log x)^{4 / 3}\right)
$$

is given by Walfisz in his book [11], posthumously published in 1963. In 1960 already, however, A. I. Saltykov proposes a proof of the better

$$
H(x)=O\left((\log x)^{2 / 3}(\log \log x)^{1+\epsilon}\right),
$$

where $\epsilon$ is an arbitrarily small positive number. His proof however contains a mistake, and once amended "only" yields Walfisz' result (1) (see the details in Section 5 below).

Saltykov's paper has been considered suspect for another reason: it relies on a theorem proved in [5], in a paper in which M.N. Korobov also makes an (as of today) unverified claim about the Riemann zeta-function (see [11], Notes on Chapter 5, p. 226). But in his work Saltykov only uses proved results of Korobov. Walfisz, who died in 1962, never mentions Saltykov's paper. It thus appears that he was unaware of its existence, as the possibility that he could have considered it suspect because of the use made in it of [5], and simply ignored it without even mentioning it (as it has been suggested), is very unlikely. Walfisz knew indeed very well Korobov's paper, and not only the few last lines of it containing the unverified claim. In fact an essential ingredient of his own proof of (1) comes from this work, as he himself acknowledges it on page 223 of [11]: "Als Quelle für $\$ 2.2$ diente die Arbeit Koroboff [1] (in this paper: [5]). Sie wurde, unter Beibehaltung aller Koroboffschen Ideen, sehr stark überarbeitet [...]".

In my joint work with U. Balakrishnan [1] I treat a class of arithmetical functions whose generating functions is of the form $\zeta(s) \zeta^{\alpha}(s+1) f(s+1)$, where $\zeta$ denotes Riemann zeta function and $f$ is some "innocuous" factor. For instance $\phi(n) / n$ is such an arithmetical function. For the related error term I generalize Walfisz' estimate (1) ([1], Theorem 2). I must admit that I have known the existence of Saltykov's paper [7] for quite a time, but that, because it is in Russian I repeatedly postponed the task of reading it, and because of its bad reputation I never referred to it in my papers. So I am very grateful to Professor A. Schinzel for twisting my arm and succeeding in making me finally read it.

My reading then was however not careful enough, and I was first (wrongly) convinced that Saltykov's proof was perfectly sound and that his result 
could be generalized, very similarly as I generalized Walfisz' in [1], thus yielding an improvement of our Theorem 2: this was announced in an "Added in proof" at the end of [1].

Also, there is a mistake in [1]. Hypothesis (h1) in Theorem 2 of this paper, stating that $\sum_{n \leq x}\left|v_{n}\right| \ll(\log x)^{\alpha}$ for some $\alpha>0$, is not sufficient to ensure the validity of this result (the last argument in the proof of Lemma 3.3 on pages 52-53 is not correct under that too weak hypothesis). Thus, Theorem 2 of [1] is not proved for a class of functions $v$ as large as stated there.

There are three objects to this paper.

(a) I first prove that the result of Theorem 2 in [1] is nevertheless true for a class of functions including all the $v$ associated to the examples treated in [1] i.e. to the error terms of the summatory functions of $(\phi(n) / n)^{-\alpha}$, $(\sigma(n) / n)^{\alpha}$ and $(\sigma(n) / \phi(n))^{\alpha / 2}$. Indeed Theorem 1 below provides the required estimate when the function $v$ satisfies hypothesis (h1) below instead of (h1) in [1]. And in fact, the proof of Theorem 2 in [1] (under the assumption of the new (h1)) can easily be amended by using Lemma 8 below in the proof of Lemma 3.3 of [1].

(b) As for Theorem 2 below, it is more widely applicable, but with a slightly weaker result than Theorem 1 . Both Theorems 1 and 2 are proved in Sections 2 and 3. Section 4 is devoted to some applications.

(c) Finally, in a short section at the end of the paper (Section 5) I give some details on Saltykov's erroneous proof.

I have a few comments on (a) and (b). In [1] our proof of Theorem 2 heavily refers to Walfisz' proof of (1) in [11]. I then chose this solution mainly because of space (our paper [1] is rather long), and this was justifiable by the availability of [11], although sometimes on the verge of inadequacy. In the present work I don't only exploit Walfisz' ideas, but also Saltykov's. On the one hand the proof of the theorems requires further generalizations of some of the auxiliary results of Walfisz and Saltykov. And on the other hand, Saltykov's paper [7] is not so easy to find, and in addition already heavily refers to Walfisz' proof - in an earlier version [10] providing a weaker estimate than (1). So I could decently not in turn heavily refer to Saltykov's paper. I finally chose to refer without proof only to auxiliary results whose original statements don't need any modification for my purpose (Lemmas A, B and C), and whose proof can easily be found in the literature. This of course means that large parts of this work are little more than a translation of large parts of [11] and [7]. I however think that a self contained proof is this time definitely required.

As I said, Korobov's results used by Saltykov are correctly proved. In any case, in the present work I use instead a later (1971) and more general result of Karatsuba's [4] with the help of which the proof can be considerably 
simplified: as soon as $M \ll x^{1-\epsilon}$ for some $\epsilon>0$, there is no need anymore to treat exponential sums of the type $\sum_{M \leq m \leq M^{\prime}} e(x / m)$ according to the size of $M$ (i.e. with a method of Weyl for the larger values of $M$, and with a method of Korobov originated from the works of Vinogradov for smaller values of $M$ ). Thus Korobov's paper [5] is not used at all in this work.

Finally, it should be mentioned that the original hypothesis (h3) in [1] is stronger than (h3) below: it requires not only that $p v(p)$, but also that $p^{2} v\left(p^{2}\right)$, be ultimately monotonic. It is the Main Lemma of Section 2 below that permits this simplification: in [1] it was only available for $k=1$; here I apply it for $k=2$ also when I prove Theorem 1 (in the proof of Lemma 11). For some applications this is appreciable, as it is sometimes not so easy to see that $p^{2} v\left(p^{2}\right)$ is ultimately monotonic (see for instance the proof of Theorem 4 in [1]).

I now state the two main results of this paper.

Theorem 1. Let $v_{n}=v(n)$ be a real multiplicative arithmetical function satisfying, for some real numbers $\alpha \geq 0, \beta \geq 0$, and $F_{r}(r \geq 0)$, and for every positive natural number $\lambda$,

$$
\begin{gathered}
\sum_{n \leq x} n\left|v_{n}\right|=x \sum_{r=0}^{\lambda+[\alpha]} F_{r}(\log x)^{\alpha-r}+O\left(x(\log x)^{-\lambda}\right) \\
\sum_{n \leq x}\left(n v_{n}\right)^{2}=O\left(x(\log x)^{\beta}\right)
\end{gathered}
$$

$p v(p)$ is an ultimately monotonic function of $p$, and $p^{\nu} v\left(p^{\nu}\right)$ is bounded for every $\nu \geq 1$.

Set $y:=x \exp \left(-(\log x)^{b}\right)$ for some positive number $b<1, t:=\log x$, and $u:=\log t=\log \log x$. Put $\psi(x):=\{x\}-1 / 2$. Then I have

$$
\sum_{n \leq y} v_{n} \psi\left(\frac{x}{n}\right)=O\left(t^{2(\alpha+1) / 3} u^{4(\alpha+1) / 3}\right)
$$

Theorem 2. Let $\Omega(n)$ denote the number of all prime divisors of $n$ ( $p$ being counted $\nu$ times if $p^{\nu}$ divides $n$ but $p^{\nu+1}$ does not). Let $v_{n}=v(n)$ be a real multiplicative arithmetical function satisfying, for some real numbers $\alpha \geq 0, \beta<1, \gamma>1, a>0, c>0$, and $F_{r}(r \geq 0)$, for every positive natural number $\lambda$, for some positive integer $K_{0} \geq 0$, and for absolute positive 
constants $K_{1}$ and $K_{2}$,

$$
\begin{gathered}
\sum_{n \leq x} n\left|v_{n}\right|=x \sum_{r=0}^{\lambda+[\alpha]} F_{r}(\log x)^{\alpha-r}+O\left(x(\log x)^{-\lambda}\right) \\
\sum_{n \leq x}\left(n v_{n}\right)^{2}=O\left(x^{2} \exp \left(-(\log x)^{c}\right)\right) \quad \text { and } \\
\sum_{p \leq x^{1 / \nu}}\left(p^{\nu} v\left(p^{\nu}\right)\right)^{2} \leq K_{1} x \quad \text { for } \nu>K_{0}
\end{gathered}
$$

(h3*) $\quad p^{\nu} v\left(p^{\nu}\right) \quad$ is an ultimately monotonic function of $p$, with

$$
\begin{gathered}
p^{\nu} v\left(p^{\nu}\right) \leq K_{2} p^{\nu-1} \quad \text { for } \nu=1,2, \cdots, K_{0} \\
\quad \text { (in particular: } p v(p) \text { is bounded) } ;
\end{gathered}
$$

$$
\sum_{n \leq x} \gamma^{\Omega(n)}\left|v_{n}\right|=O\left((\log x)^{a}\right) ;
$$

Set as before $y, t$, and $u$. Then I have

$$
\sum_{n \leq y} v_{n} \psi\left(\frac{x}{n}\right)=O\left(t^{2(\alpha+1) / 3} u^{7(\alpha+1) / 3}\right) .
$$

Remark 1. Hypothesis (h1) implies in particular that

$$
\begin{gathered}
\sum_{n \leq x} n\left|v_{n}\right| \ll x(\log x)^{\alpha}, \quad \sum_{n \leq x}\left|v_{n}\right| \ll(\log x)^{\alpha+1} \\
\text { and } \sum_{n \leq x} n^{-1}\left|v_{n}\right|=O(1)
\end{gathered}
$$

Acknowledgements. I am grateful to Professor S. Kanemitsu for transmitting to me the translation in English he made of a large part of Saltykov's paper [7].

Je remercie vivement le rapporteur, dont la lecture minutieuse m'a permis de corriger de nombreuses coquilles, petites fautes et imprécisions, et de rendre ainsi je l'espère ce travail plus agréable à lire.

\section{AN EXPONENTIAL SUM ON PRIMES.}

In this section I prove the fundamental auxiliary result just below, which I call Main Lemma. Lemmata 6 and 7 at the end of the section are also essential auxiliary results (Lemma 6, which is used for the proof of the Main Lemma, is also required for the proofs of the theorems; Lemma 7 is used for the proof of Theorem 2). I first introduce some notation. In the sequel $N$ is a real number with

$$
\exp \left(B t^{2 / 3} u^{4 / 3}\right) \leq N \leq x \exp \left(-t^{b}\right),
$$


where $B$ is some positive constant, and $t, u$, and $b$ are as in Theorem 1 . In addition I put

$$
\begin{aligned}
s & :=\log N \text { and } \\
N_{0} & :=\exp \left(\frac{s}{k_{0} \log s}\right),
\end{aligned}
$$

where $k_{0}$ is some positive constant. Also, as is customary, the symbol $p$ is used to denote prime numbers exclusively.

It should be noted (this will not be repeated below) that a number of inequalities involving $x$ and $N$ (or parameters depending on $x$ and $N$ ) I state in the sequel are not necessarily true for every positive $x$ and $N$, but only for large enough $x$ and $N$.

Main Lemma. and Let $\Lambda>0$. Then there is a choice of $B$, such that if $k$ is a positive integer and

$$
S:=\sum_{p \leq N^{\frac{1}{k}}} e\left(x / p^{k}\right)
$$

we have

$$
|S| \ll N^{\frac{1}{k}} s^{-\Lambda}
$$

Remark 2. Walfisz proves this for $k=1$ with $\Lambda=5$ (see [11], Hilfssatz 4.4.8). I note in passing that I don't claim that the constant implied by the symbol « is independent of $k$. We shall use the Main Lemma with $k=1$ and $k=2$ for the proof of Theorem 1, and with $k \leq K_{0}$ for the proof of Theorem 2. The auxiliary quantity $N_{0}$ is used in the proof, and the involved constant $k_{0}$ depends on $\Lambda$ (the value of $B$ for which the Main Lemma is satisfied will in turn depend on $k_{0}$ ). We put

$$
k_{0}:=(3 \Lambda+9) k \text {. }
$$

I first state three auxiliary lemmas, whose proofs are available in the literature.

Lemma A. If $d(n)$ denotes as usual the number of divisors of $n$ then we have

$$
\sum_{n \leq z} d(n) \ll z \log z \quad \text { and } \quad \sum_{n \leq z} d^{2}(n) \ll z(\log z)^{3}
$$

This is well known. A proof is given in [11] (see Hilfssatz 4.4.1).

Lemma B. Let $f(x)$ be real and have continuous derivatives up to the $r$-th order, where $r \geq 2$. Let $\lambda_{r} \leq f^{(r)}(x) \leq h \lambda_{r}$ for $a \leq x \leq b$, or 
$\lambda_{r} \leq-f^{(r)}(x) \leq h \lambda_{r}$ for $a \leq x \leq b$, where $b-a \geq 1$. Put $R:=2^{r-1}$. Then

$$
\sum_{a<n \leq b} e(f(n)) \ll h^{\frac{2}{R}}(b-a) \lambda_{r}^{\frac{1}{2 R-2}}+(b-a)^{1-\frac{2}{R}} \lambda_{r}^{-\frac{1}{2 R-2}} .
$$

This is also well known. A proof can be found for instance in Titchmarsh's [9]: see Theorems 5.9, 5.11 and 5.13. Or in Ivić's [2]: see Lemma 2.6. Also see Walfisz' [11], Hilfssatz 4.2.5.

Lemma C (Karatsuba, [4] Theorem 1).

Let $N$ and $P$ be integers, $P$ being positive. Let $f(x)$ be a real function having a continuous $(n+1)$-th derivative, $n \geq 2$, in the interval $N \leq x \leq$ $N+P$. Suppose that there exist positive absolute constants $c_{0}, c_{1}, c_{2}, c_{3}$ and $c_{4}$ such that $c_{0}<1, c_{1}<1$ and $c_{2}+c_{4}<c_{1}$; an integer $r$ such that $c_{0} n \leq r \leq n$; and numbers $s_{j} \geq 2(j=1, \cdots, r)$ not exceeding $k$, such that for $N \leq x \leq N+P$ the following inequalities are satisfied:

(a) $\left|f^{(n+1)}(x) /(n+1) !\right| \leq P^{-c_{1}(n+1)}$;

(b) $P^{-c_{2} s_{j}} \leq\left|f^{\left(s_{j}\right)}(x) / s_{j} !\right| \leq P^{-c_{3} s_{j}} \quad(j=1, \cdots, r)$.

Then for each positive integer $P_{1}$ not exceeding $P$, if we let

$$
S:=\sum_{N \leq x \leq N+P_{1}-1} e(f(x)),
$$

we have

$$
|S| \leq A P^{1-\gamma / n^{2}},
$$

where $A>0$ and $\gamma>0$ are absolute constants.

In the proofs of the Main Lemma and of Theorem 1 we shall use Karatsuba's theorem applied to $f(x)=z / x^{k}$.

Lemma C1. Let $k$ be a positive integer, $0<\epsilon \leq 1, z \geq Q^{k+\epsilon}$, and $\exp \left(48(k+1)^{2} / \epsilon^{2}\right)<Q \leq Q^{\prime} \leq 2 Q$, and put $\beta:=\log Q / \log z$. Then

$$
\left|\sum_{Q}^{Q^{\prime}} e\left(z / q^{k}\right)\right| \ll Q^{1-c \beta^{2}}
$$

for some absolute constant $c$.

Proof of Lemma C1. We apply Lemma C to $f(x)=z / x^{k}$ with $n=\left[48(k+1) /\left(\epsilon^{2} \beta\right)\right], N=P=Q$ and $s_{j}$ all the integer $s$ with $3 /(\epsilon \beta) \leq$ $s \leq 6 /(\epsilon \beta)$ (their number $r$ satisfying $\frac{\epsilon}{32(k+1)} n \leq r \leq n$ ), and with

$$
c_{0}=\frac{\epsilon}{32(k+1)}, \quad c_{1}=1-\frac{\epsilon^{2}}{24(k+1)}, \quad c_{2}=1-\frac{\epsilon^{2}}{12(k+1)},
$$




$$
c_{3}=1-\frac{\epsilon}{2}, \quad c_{4}=\frac{\epsilon^{2}}{25(k+1)} .
$$

We verify that conditions (a) and (b) of Lemma $\mathrm{C}$ are satisfied. We have, when $Q \leq x \leq 2 Q$,

$$
\begin{aligned}
\left|\frac{f^{(n+1)}(x)}{(n+1) !}\right| & =\prod_{\ell=1}^{n+1}\left(1+\frac{k-1}{\ell}\right) z x^{-n-k-1} \\
& \leq \exp ((k-1)(\log (n+1)+1)) Q^{\frac{1}{\beta}-\left[\frac{48(k+1)}{\epsilon^{2} \beta}\right]-k-1} \\
& \leq Q^{-\left[\frac{48(k+1)}{\epsilon^{2} \beta}\right]\left(1-\frac{\epsilon^{2}}{48(k+1)}\right)-k+\frac{(k-1)(\log (n+1)+1)}{\log Q}} \\
& \leq Q^{-(n+1)\left(1-\frac{\epsilon^{2}}{48(k+1)}\right)+\frac{n \epsilon^{2}}{48(k+1)}}<Q^{-c_{1}(n+1)}
\end{aligned}
$$

and similarly

$$
\begin{aligned}
\left|\frac{f^{\left(s_{j}\right)}(x)}{s_{j} !}\right| & =\prod_{\ell=1}^{s_{j}}\left(1+\frac{k-1}{\ell}\right) z x^{-k-s_{j}} \\
& \leq \exp \left((k-1)\left(\log \left(s_{j}\right)+1\right)\right) Q^{\frac{1}{\beta}-s_{j}-k} \\
& \leq Q^{-s_{j}\left(1-\frac{\epsilon}{3}-\frac{\epsilon^{2}}{48(k+1)}\right)-k} \leq Q^{-c_{3} s_{j}}
\end{aligned}
$$

Now note that $1 / \beta \geq \epsilon s_{j} / 6$ and $1-k \beta \geq \epsilon /(k+1)$, whence $1 / \beta-k \geq$ $\epsilon^{2} s_{j} / 6(k+1)$. We have

$$
\begin{aligned}
\left|\frac{f^{\left(s_{j}\right)}(x)}{s_{j} !}\right| & =\prod_{\ell=1}^{s_{j}}\left(1+\frac{k-1}{\ell}\right) z x^{-k-s_{j}} \geq Q^{\frac{1}{\beta}}(2 Q)^{-k-s_{j}} \\
& =Q^{\frac{1}{\beta}-k-s_{j}\left(1+\frac{\log 2}{\log Q}\right)-\frac{k \log 2}{\log Q}} \geq Q^{-s_{j}\left(-\frac{\epsilon^{2}}{6(k+1)}+1+\frac{k+1}{\log Q}\right)} \geq Q^{-c_{2} s_{j}}
\end{aligned}
$$

and the lemma is proved.

We now proceed to prove the Main Lemma.

Proof of the Main Lemma. In the three first lemmas below we first reduce the problem of estimating $S$ to the problem of estimating the more manageable expression $T(M, U)$ defined just before the statement of Lemma 3 below. If we put $P:=\prod_{p<N^{1 /(2 k)}} p$, then we have

$$
\begin{aligned}
\sum_{\substack{n \leq N \frac{1}{k} \\
(n, P)=1}} e\left(x / n^{k}\right) & =\sum_{n \leq N^{\frac{1}{k}}} e\left(x / n^{k}\right) \sum_{d \mid(n, P)} \mu(d)=\sum_{d \mid P} \mu(d) \sum_{\substack{n \leq N^{\frac{1}{k}} \\
d \mid n}} e\left(x / n^{k}\right) \\
& =\sum_{d \mid P} \mu(d) \sum_{m^{k} \leq N d^{-k}} e\left(x /(d m)^{k}\right),
\end{aligned}
$$


and

$$
\sum_{\substack{n \leq N^{\frac{1}{k}} \\(n, P)=1}} e\left(x / n^{k}\right)=e(x)+\sum_{N^{\frac{1}{2 k}<p \leq N^{\frac{1}{k}}}} e\left(x / p^{k}\right)=S+O\left(N^{\frac{1}{2 k}}\right)
$$

whence

$$
S=S_{2}-S_{1}+O\left(N^{\frac{1}{2 k}}\right)
$$

where we put

$$
S_{j}=\sum_{\substack{d m \leq N \\ d \mid P \\ \mu(d)=(-1)^{j}}} e\left(x /(d m)^{k}\right)
$$

Now we split the interval $\left[1, N^{1 / k}\right]$ in $O(\log N)=O(s)$ intervals of the form $\left[M^{1 / k}, M^{\prime}\right]$, where $M^{1 / k} \leq M^{\prime} \leq \sqrt{2} M^{1 / k} \leq \sqrt{2} N^{1 / k}$, in such a way that none of the numbers $M^{1 / k}$ is an integer. We write

$$
S_{j}=\sum_{M} S_{j}(M)
$$

where

$$
S_{j}(M):=\sum_{\substack{d \leq\left(N M^{-1}\right)^{\frac{1}{k}} \\ d \mid P \\ \mu(d)=(-1)^{j}}} \sum_{\substack{M^{\frac{1}{k}} \leq m \leq M_{1} \\ M_{1}:=\min \left(M^{\prime}, N^{\frac{1}{k}} d^{-1}\right)}} e\left(x /(d m)^{k}\right) .
$$

We dispose of the $S_{j}(M)$ with large values of $M$.

Lemma 1. For $M \geq N^{2 / 5}$ we have

$$
\left|S_{j}(M)\right| \ll N^{\frac{1}{k}} s^{-L_{1}}
$$

where $L_{1}=L_{1}(x) \rightarrow \infty$ as $x \rightarrow \infty$.

Proof. 1. First suppose that $x^{1 / 2}<N \leq x \exp \left(-t^{b}\right)$.

We apply Lemma B with $a=M^{\frac{1}{k}}$ and $b=M_{1}$. We obtain

$$
\begin{aligned}
\left|S_{j}(M)\right| \ll & M^{\frac{1}{k}} \sum_{d \leq\left(N M^{-1}\right)^{\frac{1}{k}}}\left(\frac{x}{d^{k} M^{\frac{k+r}{k}}}\right)^{\frac{1}{2 R-2}} \\
& +M^{\frac{1}{k}\left(1-\frac{2}{R}\right)} \sum_{d \leq\left(N M^{-1}\right)^{\frac{1}{k}}}\left(\frac{x}{d^{k} M^{\frac{k+r}{k}}}\right)^{-\frac{1}{2 R-2}} \\
\ll & N^{\frac{1}{k}}\left(M^{-\frac{r}{k(2 R-2)}}\left(\frac{x}{N}\right)^{\frac{1}{2 R-2}}+M^{-\frac{2}{k R}+\frac{r}{k(2 R-2)}}\left(\frac{x}{N}\right)^{-\frac{1}{2 R-2}}\right) .
\end{aligned}
$$


Put $M=\left(x N^{-1}\right)^{D}$. Note that since $x^{1 / 2}<N \leq M^{5 / 2}$ we have $M=$ $\left(x N^{-1}\right)^{D}<N^{D} \leq M^{5 D / 2}$, whence $D \geq 2 / 5$. We have

$$
N^{-\frac{1}{k}}\left|S_{j}(M)\right| \ll\left(\frac{x}{N}\right)^{\frac{1}{2 R-2}\left(1-r \frac{D}{k}\right)}+\left(\frac{x}{N}\right)^{\frac{1}{2 R-2}\left(-1+r \frac{D}{k}-\frac{2 D(2 R-2)}{k R}\right)} .
$$

Now we put $r=[k / D]+2$ and we note in passing that $2 \leq r \leq[5 k / 2]+2$. We have

$$
1-r \frac{D}{k}=1-\frac{2 D}{k}-\left[\frac{k}{D}\right] \frac{D}{k}=-\frac{D}{k}\left(2-\left\{\frac{k}{D}\right\}\right) \leq-\frac{D}{k} \leq-\frac{2}{5 k}
$$

and

$$
\left.-1+r \frac{D}{k}-\frac{2 D(2 R-2)}{k R}=\frac{D}{k}\left(-\left\{\frac{k}{D}\right\}-2+\frac{4}{R}\right)\right) .
$$

The last expression is $\leq-D / k \leq-2 /(5 k)$ if $r \geq 3$, and is $=-1$ if $r=2$ (since then $\{k / D\}=k / D)$. We recall that $N / x \leq \exp \left(-t^{b}\right)$ and we have thus

$$
N^{-\frac{1}{k}}\left|S_{j}(M)\right| \ll \exp \left(-\left(10 k 2^{5 k / 2}\right)^{-1} t^{b}\right),
$$

whence the lemma in this case.

2. There remains to consider the case where $\exp \left(B t^{2 / 3} u^{4 / 3}\right) \leq N \leq x^{1 / 2}$. We apply Lemma C1. Put $z:=x d^{-k}$ and $Q=M^{\frac{1}{k}}$. Since $d^{k} \leq N M^{-1} \leq$ $x^{3 / 10}$ and $M \leq N \leq x^{1 / 2}$, we have $z \geq x^{7 / 10} \geq M^{7 / 5} \geq Q^{7 k / 5} \geq Q^{k+2 / 5}$ and Lemma C1 holds with $\epsilon=2 / 5$. Hence

$$
\left|S_{j}(M)\right| \ll \sum_{d^{k} \leq N M^{-1}} M^{\frac{1}{k}\left(1-c(\log M)^{2}\left(\log \left(x / d^{k}\right)\right)^{-2}\right)}
$$

for some positive constant $c$. Since $\log N \ll \log M$ we have for some positive constants $c^{\prime}$ and $c^{\prime \prime}$

$$
\left|S_{j}(M)\right| \ll \sum_{d^{k} \leq N M^{-1}} M^{\frac{1}{k}\left(1-c^{\prime} \frac{\log ^{2} N}{\log ^{2} x}\right)} \ll\left(N M^{-c^{\prime} \frac{\log ^{2} N}{\log ^{2} x}}\right)^{\frac{1}{k}} \ll N^{\frac{1}{k}-c^{\prime \prime} \frac{\log ^{2} N}{\log ^{2} x}} .
$$

We conclude the proof of the lemma by noting that

$$
\exp \left(-c^{\prime \prime} \log ^{3} N / \log ^{2} x\right) \ll \exp \left(-c^{\prime \prime} B^{3} u^{4}\right) \ll t^{-L_{1}} \ll s^{-L_{1}}
$$

where $L_{1}=c^{\prime \prime} B^{3} u^{3}$.

Now we consider the case where $M \leq N^{2 / 5}$. We may write

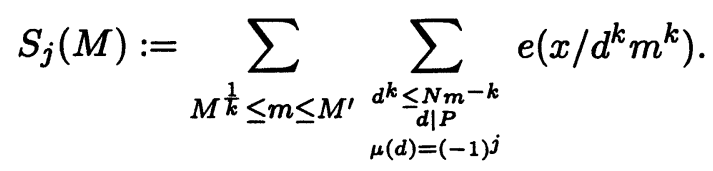

We call an integer $d$ in the expression above $\delta_{h}$ when it has exactly $h$ prime divisors $p>N_{0}$. Note that it is assumed below that $\delta_{h} \mid P$ (so that in 
particular the divisors of $\delta_{h}$ are all distinct). Let $h_{0}$ be the largest value of $h$ for $d \leq N$. Since $2^{h_{0}} \leq N$ we see that

$$
h_{0} \ll s .
$$

We have

$$
S_{j}(M)=\sum_{0 \leq h \leq h_{0}} S_{h j}(M)
$$

where

$$
S_{h j}(M):=\sum_{M^{\frac{1}{k}} \leq m \leq M^{\prime}} \sum_{\substack{\delta_{h}^{k} \leq N m^{-k} \\ \mu\left(\delta_{0}\right)=(-1)^{j} \\ \delta_{h} \mid P}} e\left(x / \delta_{h}^{k} m^{k}\right) .
$$

We dispose of $S_{0 j}(M)$.

Lemma 2. For $M \leq N^{2 / 5}$ we have

$$
\left|S_{0 j}(M)\right| \ll N^{\frac{1}{k}} s^{-\frac{k_{0}}{3 k}+1} .
$$

Proof. Let $L_{2}:=-k_{0} /(3 k)+1$ and suppose that $\delta_{0}>\left(N M^{-1}\right)^{\frac{1}{k}} s^{-L_{2}}$. If $\ell$ denotes the number of (necessarily distinct) prime divisors of $\delta_{0}$ (each of which, by definition of $\delta_{0}$, doesn't exceed $N_{0}$ ), we have

$$
N_{0}^{\ell} \geq \delta_{0}>\left(N M^{-1}\right)^{\frac{1}{k}} s^{-L_{2}} \geq N^{\frac{3}{5 k}} s^{-L_{2}} \geq N^{\frac{1}{2 k}}
$$

whence

Hence

$$
\frac{\ell s}{k_{0} \log s} \geq \frac{s}{2 k} \quad \text { or } \quad \ell \geq \frac{k_{0}}{2 k} \log s .
$$

$$
d\left(\delta_{0}\right) \geq 2^{\ell} \geq \exp \left(\frac{k_{0} \log 2}{2 k} \log s\right) \geq s^{\frac{k_{0}}{3 k}}
$$

Thus by Lemma A we have

$$
\begin{aligned}
\left|S_{0 j}(M)\right| & \ll \sum_{M^{\frac{1}{k}} \leq m \leq M^{\prime}}\left(\sum_{n \leq\left(N M^{-1}\right)^{\frac{1}{k}} s^{-L_{2}}} 1+s^{-\frac{k_{0}}{3 k}} \sum_{n \leq\left(N M^{-1}\right)^{\frac{1}{k}}} d(n)\right) \\
& \ll N^{\frac{1}{k}} s^{-L_{2}}+N^{\frac{1}{k}} s^{-\frac{k_{0}}{3 k}+1},
\end{aligned}
$$

and the lemma is proved.

Now when $0<h \leq h_{0}$ we write

$$
\begin{aligned}
T_{h j}(M) & :=\sum_{\substack{M^{\frac{1}{k}} \leq m \leq M^{\prime} \\
\begin{array}{c}
(p q)^{k} \leq N m^{-k} \\
N_{0}<p<N^{1 /(2 k)} \\
q=\delta_{h-1} \\
\mu(q)=(-1)^{j-1}
\end{array}}} e\left(\frac{x}{(p q m)^{k}}\right) \\
& =: T_{h j 1}(M)+T_{h j 2}(M),
\end{aligned}
$$


where $T_{h j 2}(M)$ is restricted to the terms with $p \mid q$ and $T_{h j 1}(M)$ to the others. (Note that for instance $T_{1 j 2}(M)$ is empty). Putting $q=p r$ we see that

$$
\begin{aligned}
\left|T_{h j 2}(M)\right| & \ll \sum_{M^{\frac{1}{k}} \leq m \leq M^{\prime}} \sum_{N_{0}<p \leq N} \sum_{r^{\frac{1}{2 k}}} \sum_{r^{k} \leq N m^{-k} p^{-2 k}} 1 \\
& \ll N^{\frac{1}{k}} \sum_{M^{\frac{1}{k} \leq m \leq 2 M^{\frac{1}{k}}} m^{-1} \sum_{n>N_{0}} n^{-2}} \\
& \ll N^{\frac{1}{k}} N_{0}^{-1} \ll N^{\frac{1}{k}} s^{-L_{3}}
\end{aligned}
$$

where $L_{3}=s /\left(k_{0} \log ^{2} s\right)$. Since $T_{h j 1}(M)=h S_{h j}(M)$ we have

(6) $\quad S_{h j}(M)=h^{-1} T_{h j}(M)-h^{-1} T_{h j 2}(M)=h^{-1} T_{h j}(M)+O\left(N^{\frac{1}{k}} s^{-L_{3}}\right)$.

And now we split the summation interval $N_{0}<p \leq N^{1 /(k+1)}$ of the inside sum of $T_{h j}(M)$ in $O(s)$ intervals of the form $U^{\frac{1}{k}} \leq p \leq U^{\prime}$, where $N_{0}<U^{\frac{1}{k}} \leq U^{\prime} \leq \sqrt{2} U^{\frac{1}{k}} \leq \sqrt{2} N^{1 /(2 k)}$ and we write

$$
T_{h j}(M)=\sum_{U} T_{h j}(M, U)
$$

where

Finally we define, for $k_{2}>0$,

$$
T_{h j}(M, U):=\sum_{\substack{M^{\frac{1}{k}} \leq m \leq M^{\prime} \\ U^{\frac{1}{k}} \leq p \leq U^{\prime}}} \sum_{\substack{q^{k} \leq N m-p_{p}-k \\ q=\delta_{h-1} \\ \mu(q)=(-1)^{j-1}}} e\left(x / m^{k} p^{k} q^{k}\right) .
$$

$$
\begin{aligned}
& T(M, U)=T\left(M, U, V, V^{\prime}, k_{2}\right)
\end{aligned}
$$

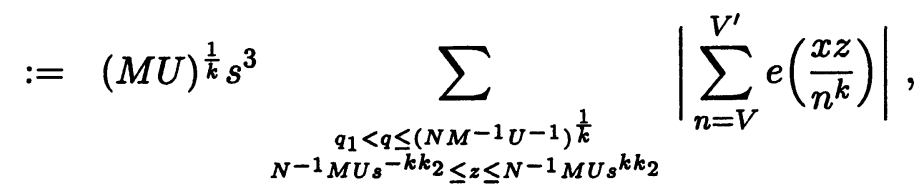

where $z:=q_{1}^{-k}-q^{-k}$ and $(M U)^{\frac{1}{k}} \leq V=V\left(q, q_{1}\right) \leq V^{\prime}=V^{\prime}\left(q, q_{1}\right) \leq$ $2(M U)^{\frac{1}{k}}$, and we prove

Lemma 3. For $M \leq N^{2 / 5}$ and $U$ as in the definition of $T_{h j}(M, U)$ we have

$$
\left|T_{h j}(M, U)\right|^{2} \ll T(M, U)+N^{\frac{2}{k}} s^{3-k_{2}} .
$$

Proof. Recall that $M^{\prime} U^{\prime} \leq \sqrt{2} M^{\frac{1}{k}} \sqrt{2} U^{\frac{1}{k}}=2(M U)^{\frac{1}{k}}$. If we write

$$
d^{\prime}(n)=\sum_{\substack{m p=n \\ M^{\frac{1}{k}} \leq m \leq M^{\prime} \\ U^{\frac{1}{k}} \leq p \leq U^{\prime}}} 1
$$


we have

$$
T_{h j}(M, U)=\sum_{(M U)^{\frac{1}{k}} \leq n \leq M^{\prime} U^{\prime}} d^{\prime}(n) \sum_{q^{k} \leq N n^{-k}}^{\prime} e\left(x / n^{k} q^{k}\right),
$$

where $\sum^{\prime}$ means that $q$ satisfies $q=\delta_{h-1}$ and $\mu(q)=(-1)^{j-1}$. Thus

$$
\left|T_{h j}(M, U)\right| \leq \sum_{(M U)^{\frac{1}{k}} \leq n \leq M^{\prime} U^{\prime}} d(n)\left|\sum_{q^{k} \leq N n^{-k}}^{\prime} e\left(x / n^{k} q^{k}\right)\right|
$$

and

$$
\left|T_{h j}(M, U)\right|^{2} \leq \sum_{(M U)^{\frac{1}{k}} \leq n \leq M^{\prime} U^{\prime}} d^{2}(n) \sum_{(M U)^{\frac{1}{k}} \leq n \leq M^{\prime} U^{\prime}}\left|\sum_{q^{k} \leq N n^{-k}}^{\prime} e\left(x / n^{k} q^{k}\right)\right|^{2} .
$$

Hence a use of Lemma A yields

$$
\left|T_{h j}(M, U)\right|^{2} \ll(M U)^{\frac{1}{k}} s^{3} \sum_{(M U)^{\frac{1}{k}} \leq n \leq M^{\prime} U^{\prime}}\left|\sum_{q^{k} \leq N n^{-k}}^{\prime} e\left(x / n^{k} q^{k}\right)\right|^{2}
$$

Since we have

$$
\begin{aligned}
\left|\sum_{q^{k} \leq N n^{-k}}^{\prime} e\left(x / n^{k} q^{k}\right)\right|^{2} & =\sum_{q^{k} \leq N n^{-k}}^{\prime} e\left(x / n^{k} q^{k}\right) \sum_{q^{k} \leq N n^{-k}}^{\prime} e\left(-x / n^{k} q^{k}\right) \\
& =\sum_{q^{k}, q_{1}^{k} \leq N n^{-k}}^{\prime} e\left(x z / n^{k}\right),
\end{aligned}
$$

the last estimate can be rewritten as

$$
\begin{aligned}
& \left|T_{h j}(M, U)\right|^{2} \ll(M U)^{\frac{1}{k}} s^{3} \sum_{(M U)^{\frac{1}{k} \leq n \leq M^{\prime} U^{\prime}}} \sum_{q^{k}, q_{1}^{k} \leq N n^{-k}}^{\prime} e\left(x z / n^{k}\right) \\
& \ll(M U)^{\frac{1}{k}} s^{3} \sum_{q^{k}, q_{1}^{k} \leq N M^{-1} U^{-1}}^{\prime} \sum_{(M U)^{\frac{1}{k}} \leq n \leq \min \left(M^{\prime} U^{\prime}, N^{\frac{1}{k}} q^{-1}, N^{\frac{1}{k}}\right.} e\left(x z / n_{1}^{k}\right)
\end{aligned}
$$

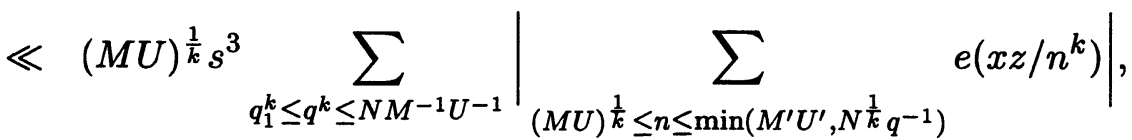

where we now let $q$ and $q_{1}$ take all positive integral values not exceeding $\left(N M^{-1} U^{-1}\right)^{1 / k}$. To this last estimate the terms with $q=q_{1}$ contribute at most a $O$ of

$$
\begin{aligned}
(M U)^{\frac{1}{k}} s^{3} \sum_{q^{k} \leq N M^{-1} U^{-1}} \sum_{(M U)^{\frac{1}{k}} \leq n \leq 2(M U)^{\frac{1}{k}}} 1 & \ll(N M U)^{\frac{1}{k}} s^{3} \\
& \ll N^{\left(1+\frac{2}{5}+\frac{1}{2}\right) \frac{1}{k}} s^{3} \ll N^{\frac{2}{k}} s^{3-k_{2}},
\end{aligned}
$$


whence

$$
\left|T_{h j}(M, U)\right|^{2} \ll(M U)^{\frac{1}{k}} s^{3} \sum_{q_{1}^{k}<q^{k} \leq N M^{-1} U^{-1}}\left|\sum_{n=V}^{V^{\prime}} e\left(x z / n^{k}\right)\right|+N^{\frac{2}{k}} s^{3-k_{2}}
$$

where $V$ and $V^{\prime}$ are as in the definition of $T(M, U)$ above. Now we have

$$
(M U)^{\frac{1}{k}} s^{3} \sum_{\substack{q \leq\left(N M^{-1} U^{-1}\right)^{\frac{1}{k}} \\ q_{1}<\left(N M^{-1} U^{-1}\right)^{\frac{1}{k}} s^{-k_{2}}}}(M U)^{\frac{1}{k}} \ll N^{\frac{2}{k}} s^{3-k_{2}},
$$

and thus

$$
\begin{gathered}
\left|T_{h j}(M, U)\right|^{2} \\
\ll(M U)^{\frac{1}{k}} s^{3} \sum_{\left(N M^{-1} U^{-1}\right)^{\frac{1}{k}} s^{-k_{2}} \leq q_{1}<q \leq\left(N M^{-1} U^{-1}\right)^{\frac{1}{k}}}\left|\sum_{n=V}^{V^{\prime}} e\left(x z / n^{k}\right)\right|+N^{\frac{2}{k}} s^{3-k_{2}} .
\end{gathered}
$$

Moreover we have

$$
\begin{aligned}
(M U)^{\frac{1}{k}} s^{3} & \sum_{\substack{q^{k} \leq N M^{-1} U^{-1} \\
0<q^{k}-q_{1}^{k} \leq N M^{-1} U^{-1} s^{-k k_{2}}}}(M U)^{\frac{1}{k}} \\
& \ll(M U)^{\frac{1}{k}} s^{3}\left(N M^{-1} U^{-1}\right)^{\frac{2}{k}} s^{-k_{2}}(M U)^{\frac{1}{k}} \ll N^{\frac{2}{k}} s^{3-k_{2}},
\end{aligned}
$$

whence

$$
\begin{aligned}
& \left|T_{h j}(M, U)\right|^{2} \\
& \ll(M U)^{\frac{1}{k}} s^{3} \sum_{\substack{\left(N M^{-1} U^{-1}\right)^{\frac{1}{k} s^{-k}} \\
q^{k}-q_{1}^{k}>N M^{-1} U^{-1} s^{-k k_{2}}}}\left|\sum_{n=V}^{V^{\prime}} e\left(x z / n^{k}\right)\right|+N^{\frac{2}{k}} s^{3-k_{2}} .
\end{aligned}
$$

Now when the conditions $\left(N M^{-1} U^{-1}\right)^{\frac{1}{k}} s^{-k_{2}} \leq q_{1}<q \leq\left(N M^{-1} U^{-1}\right)^{\frac{1}{k}}$ and $q^{k}-q_{1}^{k}>N M^{-1} U^{-1} s^{-k k_{2}}$ are satisfied we also have $N^{-1} M U s^{-k k_{2}} \leq$ $z \leq N^{-1} M U s^{k k_{2}}$, and the lemma is proved.

To complete the proof of the Main Lemma, there thus remains to estimate $T(M, U)$ when $M \leq N^{2 / 5}$. We consider a wider class of functions. 
Definition. For $k_{2}>0, z:=q_{1}^{-k}-q^{-k}$, and $v$ an arithmetical function satisfying (h1) of Theorems 1 and 2 let

$$
\begin{aligned}
& T(v, M, U)=T\left(v, M, U, V, V^{\prime}, k_{2}, k\right)
\end{aligned}
$$

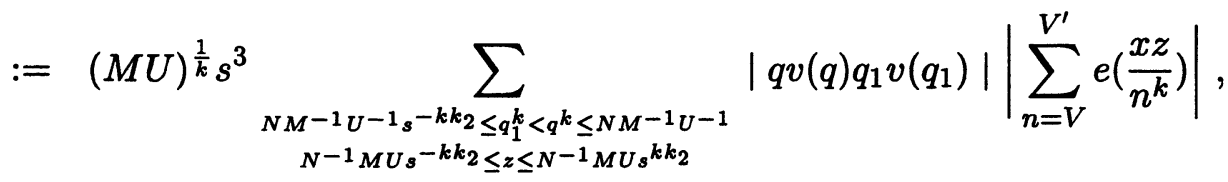

where $M, U, V$ and $V^{\prime}$ are assumed to satisfy

$$
\begin{aligned}
& \text { either } M \leq N^{2 / 5} \text { and } N_{0}<U \leq N^{1 / 2} \\
& \quad \text { or } M=1 \text { and } N_{0}<U \leq N N_{0}^{-1} \text {, } \\
& \text { and }(M U)^{\frac{1}{k}} \leq V=V\left(q, q_{1}\right) \leq V^{\prime}=V^{\prime}\left(q, q_{1}\right) \leq 2(M U)^{\frac{1}{k}} .
\end{aligned}
$$

Remark 3. We shall prove with the three next lemmas somewhat more than we need just now, i.e. that for each choice of the positive constants $k_{0}$ and $k_{2}$ and for every $\Lambda>0$ there is a choice of $B$ ensuring that

$$
T(v, M, U) \ll N^{\frac{2}{k}} s^{-L} .
$$

This will of course be used in order to complete the proof of the Main Lemma (with simply $v(q)=1 / q$ ). But it will also be used in the next section for the proof of the theorems, with $k=1$.

Lemma 4. Write $z=N^{-1} M U s^{k k_{2} \lambda}$ for some $\lambda$ with $-1 \leq \lambda \leq 1$ and define $\beta=\beta(M U, \lambda)$ with $M U=(x z)^{\beta}$. Now let $A\left(\log \left(x N^{-1} s^{k k_{2}}\right)\right)^{-1 / 3} u^{1 / 3} \leq$ $\beta \leq 3 / 4$ where $A$ is some positive constant. Let $L_{5}>0$. Then there is a choice of $A$ for which

$$
T(v, M, U) \ll N^{\frac{2}{k}} s^{-L_{5}} .
$$

Proof. Since $M U=\left(x N^{-1} s^{k k_{2} \lambda} M U\right)^{\beta}$ we have $M U=\left(x N^{-1} s^{k k_{2} \lambda}\right)^{\beta /(1-\beta)}$ $>\left(x N^{-1} s^{k k_{2} \lambda}\right)^{\beta}$, whence (by using $\log \left(x N^{-1} s^{-k k_{2}}\right)=\log \left(x N^{-1} s^{k k_{2}}\right)(1+$ $o(1)))$

$$
\log (M U)>\frac{A}{2}\left(\log \left(x N^{-1} s^{k k_{2}}\right)\right)^{\frac{2}{3}} u^{\frac{1}{3}}
$$

And since $x z=(M U)^{1 / \beta} \geq(M U)^{4 / 3} \geq(V / 2)^{4 k / 3} \geq(V / 2)^{k+1 / 3} \geq V^{k+1 / 4}$ we may apply Lemma C1 with $\epsilon=1 / 4, Q=V$, and $x z$ instead of $z$. We 
have, for some positive constant $c$,

$$
\begin{aligned}
\left|\sum_{V}^{V^{\prime}} e\left(x z / n^{k}\right)\right| & \ll(M U)^{\frac{1}{k}\left(1-c \beta^{2}\right)} \\
& \ll(M U)^{\frac{1}{k}} \exp \left(\log (M U)\left(-\frac{c A^{2}}{k} \log ^{-2 / 3}\left(x N^{-1} s^{k k_{2}}\right) u^{2 / 3}\right)\right) \\
& \ll(M U)^{\frac{1}{k}} \exp \left(-\frac{c A^{3}}{2 k} u\right) \ll(M U)^{\frac{1}{k}} s^{-L_{6}}
\end{aligned}
$$

if $A^{3} \geq 2 k L_{6} / c$. So if we choose $L_{6} \geq L_{5}+3+2 \alpha$ the lemma follows.

Lemma 5. If $M U \geq x N^{-1}$ then

$$
T(v, M, U) \ll N^{\frac{2}{k}} s^{-L_{7}}
$$

for some $L_{7}(x) \rightarrow \infty$ as $x \rightarrow \infty$.

Proof. We apply Lemma B, with $f(n)=x z / n^{k}, a=V-1$, and $b=V^{\prime}$.

We have

$$
\begin{aligned}
& \left|\sum_{V} e\left(\frac{x z}{n^{k}}\right)\right| \ll(M U)^{\frac{1}{k}}\left(\frac{x z}{(M U)^{\frac{k+r}{k}}}\right)^{\frac{1}{2 R-2}}+(M U)^{\frac{1}{k}\left(1-\frac{2}{R}\right)}\left(\frac{x z}{(M U)^{\frac{k+r}{k}}}\right)^{-\frac{1}{2 R-2}} \\
\ll & (M U)^{\frac{1}{k}\left(1-\frac{k+r}{2 R-2}\right)}\left(x N^{-1} M U s^{k k_{2}}\right)^{\frac{1}{2 R-2}}+(M U)^{\frac{1}{k}\left(1-\frac{2}{R}+\frac{k+r}{2 R-2}\right)}\left(x N^{-1} M U s^{-k k_{2}}\right)^{-\frac{1}{2 R-2}} \\
\ll & \left(x^{\frac{1}{2 R-2}} N^{-\frac{1}{2 R-2}}(M U)^{\frac{1}{k}\left(1-\frac{r}{2 R-2}\right)}+x^{-\frac{1}{2 R-2}} N^{\frac{1}{2 R-2}}(M U)^{\frac{1}{k}\left(1-\frac{2}{R}+\frac{r}{2 R-2}\right)}\right)^{\frac{k k_{2}}{2 R-2}},
\end{aligned}
$$

whence

$$
\begin{aligned}
T(v, M, U) \ll s^{\frac{k k_{2}}{2 R-2}+2 \alpha+3}\left(x^{\frac{1}{2 R-2}} N^{\frac{2}{k}-\frac{1}{2 R-2}}(M U)^{-\frac{r / k}{2 R-2}}\right. \\
\quad+x^{\left.-\frac{1}{2 R-2} N^{\frac{2}{k}+\frac{1}{2 R-2}}(M U)^{\frac{r / k}{2 R-2}-\frac{2 / k}{R}}\right)}
\end{aligned}
$$

or, if we put $M U=\left(x N^{-1}\right)^{D}(D \geq 1)$,

$$
\begin{aligned}
& T(v, M, U) s^{-\frac{k k_{2}}{2 R-2}-2 \alpha-3} N^{-\frac{2}{k}} \\
\ll & \left(\frac{x}{N}\right)^{\frac{1}{2 R-2}}(M U)^{-\frac{r / k}{2 R-2}}+\left(\frac{x}{N}\right)^{-\frac{1}{2 R-2}}(M U)^{\frac{r / k}{2 R-2}-\frac{2 / k}{R}} \\
= & \left(\frac{x}{N}\right)^{\frac{1}{2 R-2}\left(1-\frac{r D}{k}\right)}+\left(\frac{x}{N}\right)^{\frac{1}{2 R-2}\left(-1+\frac{r D}{k}-\frac{2(2 R-2) D}{k R}\right)}
\end{aligned}
$$

Now if we set $r=[k / D]+2$ as in the proof of Lemma 1 , then we have $2 \leq r \leq k+2$ and $2 R-2<2^{k+2}$, whence

$$
\frac{1}{2 R-2}\left(1-\frac{r D}{k}\right)<-\frac{1}{k 2^{k+2}}
$$

and

$$
\frac{1}{2 R-2}\left(-1+\frac{r D}{k}-\frac{2(2 R-2) D}{k R}\right)=-\frac{D}{k(2 R-2)}\left(\left\{\frac{k}{D}\right\}+2-\frac{4}{R}\right) \leq-\frac{1}{k 2^{k+2}} \text {. }
$$


(As in the proof of Lemma 1 the last estimate is obtained by considering separately the cases $r \geq 3$ and $r=2$ ). Hence we have

$$
T(v, M, U) s^{-\frac{k k_{2}}{2 R-2}-2 \alpha-3} N^{-\frac{2}{k}} \ll \exp \left(-\frac{t^{b}}{k 2^{k+2}}\right),
$$

and the lemma is proved.

Lemma 6. Let $L>0$ and choose the constant $A$ sufficiently large to ensure that Lemma 4 holds for $L_{5}=L$. Put $B=3 A k_{0}$. Then

$$
T(v, M, U) \ll N^{\frac{2}{k}} s^{-L} .
$$

Proof. 1. By Lemma 5, Lemma 6 is true when $M U \geq x N^{-1}$. By Lemma 4 it is also true for $M U=\left(x N^{-1} s^{k k_{2} \lambda}\right)^{\beta /(1-\beta)}$, when $A\left(\log \left(x N^{-1} s^{k k_{2}}\right)\right)^{-1 / 3} u^{1 / 3}$ $\leq \beta \leq 3 / 4$, and thus when $2 A\left(\log \left(x N^{-1} s^{k k_{2}}\right)\right)^{-1 / 3} u^{1 / 3} \leq \beta_{2} \leq 3$ if $\beta_{2}:=$ $\beta /(1-\beta)$. Hence it is true for

$$
M U \geq\left(x N^{-1} s^{-k k_{2}}\right)^{2 A\left(\log \left(x N^{-1} s^{k k_{2}}\right)\right)^{-1 / 3} u^{1 / 3}}
$$

Since on the other hand $M U \geq N_{0}$, in order to conclude the proof it is sufficient to ensure that

$$
\left(x N^{-1} s^{-k k_{2}}\right)^{2 A\left(\log \left(x N^{-1} s^{k k_{2}}\right)\right)^{-1 / 3} u^{1 / 3}} \leq N_{0}=\exp \left(\frac{s}{k_{0} \log s}\right) .
$$

Since finally $\log \left(x N^{-1} s^{k k_{2}}\right)=\log \left(x N^{-1}\right)(1+o(1))$ the last estimate will in turn be verified if

$$
3 A\left(\log \left(x N^{-1}\right)\right)^{\frac{2}{3}} u^{\frac{1}{3}} \leq \frac{s}{k_{0} \log s} .
$$

By hypothesis we have $t \geq s \geq B t^{2 / 3} u^{4 / 3}$, so that

$$
3 A\left(\log \left(x N^{-1}\right)\right)^{\frac{2}{3}} u^{\frac{1}{3}} \leq 3 A t^{\frac{2}{3}} u^{\frac{1}{3}}=\frac{B}{k_{0}} t^{\frac{2}{3}} u^{\frac{1}{3}} \leq \frac{s}{k_{0} \log s},
$$

and Lemma 6 is proved.

We are now in position to conclude the proof of the Main Lemma. We use Lemma 6 with the arithmetical function $v(n)=1 / n$. From Lemma 3 we see that (for $0<h \leq h_{0}$ )

$$
\left|T_{h j}(M, U)\right| \ll N^{\frac{1}{k}} s^{\frac{3}{2}-\frac{k_{2}}{2}} \quad \text { when } \quad M \leq N^{\frac{2}{5}} .
$$

This implies with (7), (6) and Lemma 2 that (for $0 \leq h \leq h_{0}$ )

$$
\left|S_{h j}(M)\right| \ll N^{\frac{1}{k}}\left(s^{\frac{5}{2}-\frac{k_{2}}{2}}+s^{1-\frac{k_{0}}{3 k}}\right) \quad \text { when } \quad M \leq N^{\frac{2}{5}} .
$$

Now with (4), (5) and Lemma 1 we see that

$$
\left|S_{j}(M)\right| \ll N^{\frac{1}{k}}\left(s^{\frac{7}{2}-\frac{k_{2}}{2}}+s^{2-\frac{k_{0}}{3 k}}\right),
$$


and finally with (3) and (2) that, if we choose $k_{2} \geq 3+2 k_{0} /(3 k)$,

$$
|S| \ll N^{\frac{1}{k}}\left(s^{\frac{9}{2}-\frac{k_{2}}{2}}+s^{3-\frac{k_{0}}{3 k}}\right) \ll N^{\frac{1}{k}} s^{3-\frac{k_{0}}{3 k}}
$$

This concludes the proof of the Main Lemma.

Remark 4. Given $\Lambda>0$ we may choose the various constants involved in the proof as follows. As is mentioned at the beginning of the section we set $k_{0}=(3 \Lambda+9) k$. Then we put $k_{2}=3+2 k_{0} /(3 k)$, and we choose $A$ large enough to ensure that Lemma 4 is satisfied with $L_{5}=-3+k_{2}$. Finally we set $B=3 A k_{0}$.

Remark 5. Given $\Lambda>0$, we shall require in the next section the Main Lemma to simultaneously hold for $k=1,2, \ldots, K_{0}$. This can be ensured by setting $k_{0}=(3 \Lambda+9) K_{0}, k_{2}=3+2 k_{0} / 3, A$ large enough to ensure that Lemma 4 is satisfied with $L_{5}=-3+k_{2}$ for $k=1,2, \ldots, K_{0}$, and $B=3 A k_{0}$.

We conclude this section by an auxiliary result required for the proof of Theorem 2.

\section{Lemma 7. Let}

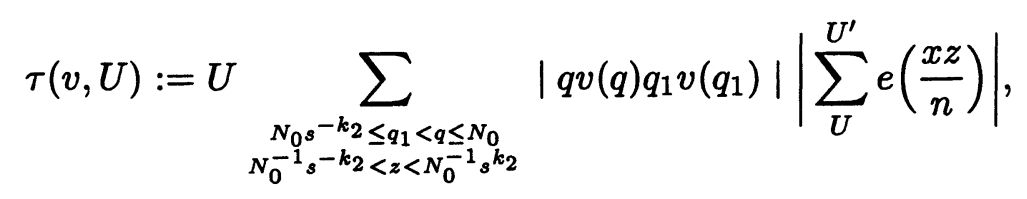

where $N_{0} \leq U \leq U^{\prime} \leq 2 U \leq 2 N N_{0}^{-1}$, and let $L>0$. Then, provided the constant $B$ (with $B t^{2 / 3} u^{4 / 3} \leq s$ ) is chosen large enough we have

$$
\tau(v, U) \ll N^{2} s^{-L}
$$

Proof. The proof is very similar to that of Lemma 6. Briefly, it goes as follows.

First, as in Lemma 4, we show that $\tau(v, U) \ll N^{2} s^{-L}$ if $U=(x z)^{\beta}$ with the condition $A \log \left(x N_{0}^{-1} s^{k_{2}}\right)^{-1 / 3} u^{1 / 3} \leq \beta \leq 3 / 4$ (provided $A$ is chosen large enough). For this we apply Lemma C1 with $\epsilon=1 / 4, Q=U$ and $x z$ instead of $z$.

Then, as in Lemma 5, we show that If $U \geq\left(x N_{0}^{-1} s^{-k_{2}}\right)^{3 / 4}$ then $\tau(v, U) \ll$ $N^{2} s^{-L_{7}^{\prime}}$ for some $L_{7}^{\prime}(x) \rightarrow \infty$ as $x \rightarrow \infty$. For this we apply Lemma B with 
$r=2$ : we obtain, by also using $U \leq 2 N N_{0}^{-1} \ll x N_{0}^{-1} \exp \left(-t^{b}\right)$ and Re$\operatorname{mark} 1$,

$$
\begin{aligned}
\tau(v, U) & \ll U N_{0}^{2}\left(U\left(\frac{x z}{U^{3}}\right)^{\frac{1}{2}}+U^{1-1}\left(\frac{x z}{U^{3}}\right)^{-\frac{1}{2}}\right) s^{2 \alpha} \\
& \ll N^{2}\left(\left(\frac{x}{N_{0}}\right)^{\frac{1}{2}} U^{-\frac{3}{2}}+\left(\frac{x}{N_{0}}\right)^{-\frac{1}{2}} U^{\frac{1}{2}}\right) s^{2 \alpha+k_{2} / 2} \\
& \ll\left(\left(\frac{x}{N_{0}}\right)^{-\frac{1}{2}}+\exp \left(-\frac{1}{2} t^{b}\right)\right) s^{2 \alpha+k_{2} / 2}
\end{aligned}
$$

Finally we conclude the proof as in that of Lemma 6, by ensuring that

$$
2 A\left(\log \left(x N_{0}^{-1}\right)\right)^{2 / 3} u^{1 / 3} \leq \frac{s}{k_{0} \log s} .
$$

Note that for this it is sufficient to choose $B \geq 2 A k_{0}$ : thus $B$ as in Lemma 6 is satisfactory.

\section{PROOF OF THE THEOREMS.}

The proofs of both theorems are very similar and are presented simultaneously below. Only a few estimates are obtained differently if hypotheses $\left(\mathrm{h} 2^{*}\right),\left(\mathrm{h} 3^{*}\right)$ and $(\mathrm{h} 4)$ of Theorem 2 are assumed instead of (h2) and (h3) of Theorem 1. For both proofs we shall need

Lemma 8. If $v$ satisfies hypothesis (h1) and if $y \leq x(\log x)^{-\lambda}$ then

$$
\sum_{x<n \leq x+y} n|v(n)| \ll x(\log x)^{-\lambda+\alpha} .
$$

Proof. If $\alpha \geq \beta>0$ and $\beta^{\prime}:=[\beta]+1$ we have

$$
\begin{aligned}
(\log (x+y))^{\beta}-(\log x)^{\beta} & \leq(\log (x+y))^{\beta^{\prime}}-(\log x)^{\beta^{\prime}} \\
& \leq \beta^{\prime} \log (1+y / x)(\log (x+y))^{\beta^{\prime}-1} \\
& \leq \beta^{\prime} \frac{y}{x}(\log (2 x))^{\beta^{\prime}-1} \leq \beta^{\prime}(\log x)^{-\lambda}(\log (2 x))^{\beta^{\prime}-1} \\
& \leq \beta^{\prime} 2^{\beta}(\log x)^{-\lambda+\beta}
\end{aligned}
$$

and if $-\lambda-1<\beta<0$ and $\beta^{\prime}:=[-\beta]+1$ we have

$$
\begin{aligned}
\left|(\log (x+y))^{\beta}-(\log x)^{\beta}\right| & \leq(\log x)^{2 \beta}\left((\log (x+y))^{-\beta}-(\log x)^{-\beta}\right) \\
& \leq \beta^{\prime}(\log x)^{-\lambda+2 \beta}(\log (2 x))^{\beta^{\prime}-1} \\
& \leq \beta^{\prime} 2^{-\beta}(\log x)^{-\lambda+\beta} .
\end{aligned}
$$


Hence

$$
\begin{aligned}
\sum_{x<n \leq x+y} n|v(n)|= & x \sum_{r=0}^{\lambda+[\alpha]} F_{r}\left((\log (x+y))^{\alpha-r}-(\log x)^{\alpha-r}\right) \\
& +y \sum_{r=0}^{\lambda+[\alpha]} F_{r}(\log (x+y))^{\alpha-r}+O\left(x(\log x)^{-\lambda}\right) \\
\ll & x \sum_{r=0}^{\lambda+[\alpha]}(\log x)^{-\lambda+\alpha-r}+x(\log x)^{-\lambda+\alpha}
\end{aligned}
$$

and the lemma.

We let similarly as in Section $2 N \leq x \exp \left(-t^{b}\right)$ be a real number and put as before $s:=\log N$ and $t, u$ and $b$ as in Theorems 1 and 2. We will make use as before of an auxiliary quantity

$$
N_{0}:=\exp \left(\frac{s}{k_{0} \log s}\right) .
$$

and shall assume from now on, for the proof of Theorem $\vartheta(\vartheta=1$ or 2$)$, that $N$ satisfies

$$
\exp \left(B^{\prime} t^{\frac{2}{3}} u^{\frac{1}{3}+\vartheta}\right) \leq N \leq x \exp \left(-t^{b}\right)
$$

with

$$
\begin{aligned}
& k_{0} \geq 16+2 \beta \quad \text { and } \quad B^{\prime} \geq 4 B \quad \text { if } \vartheta=1 \\
& k_{0} \geq(a+4) 2 / \log \gamma \quad \text { and } \quad B^{\prime} \geq 3 k_{0} B \quad \text { if } \vartheta=2
\end{aligned}
$$

where $B$ is large enough to ensure that the Main Lemma holds for $\Lambda=\alpha+5$, for $k=1$ and 2 if $\vartheta=1$, and for $k=1,2, \ldots, K_{0}$ if $\vartheta=2$. In order to simplify the notation we put $K_{0}=2$ in case $\vartheta=1$. We also ask that $B$ be large enough to ensure that Lemma 6 with $k=M=1$ and Lemma 7 both hold for $L=12$. The first condition on $B$ can be satisfied with the use of an auxiliary quantity such as $N_{0}$ with $k_{0} \geq(3 \alpha+24) K_{0}$, and of an auxiliary constant $k_{2}$ (as in the definition of $T(v, M, U)$ ) with $k_{2} \geq 3+2 k_{0} / 3 \geq(2 \alpha+16) K_{0}+3$ in the proof of the Main Lemma: see Remarks 4 and 5; the second condition, however, can be achieved with any (positive) choice of the auxiliary numbers $k_{0}$ and $k_{2}$. On the other hand we shall see below that the proof requires to choose $k_{2} \geq 24+4 \alpha$ (see the last lines of this section). We may thus set

$$
k_{0}=(3 \alpha+24) K_{0} \quad \text { and } \quad k_{2}=(4 \alpha+24) K_{0} .
$$


We prove the theorems for $x$ an integer; if $x$ is not an integer the results then follow from (h1) (see Remark 1), since

$$
\sum_{n \leq y} v_{n}\left(\psi\left(\frac{x}{n}\right)-\psi\left(\frac{[x]}{n}\right)\right)=O\left(\sum_{n \leq y} \frac{\left|v_{n}\right|}{n}\right)=O(1) .
$$

Now if $w:=\exp \left(B^{\prime} t^{\frac{2}{3}} u^{\frac{1}{3}+\vartheta}\right)$ it is an immediate consequence of (h1) (also see Remark 1) that

$$
\sum_{n \leq w} v_{n} \psi\left(\frac{x}{n}\right)=O\left(t^{\frac{2(\alpha+1)}{3}} u^{(\alpha+1)\left(\frac{1}{3}+\vartheta\right)}\right) .
$$

We shall prove that

$$
\sum_{w \leq n \leq y} v_{n} \psi\left(\frac{x}{n}\right)=O(1)
$$

whence the theorems. The last sum consists in $O(t)$ sums of the type $\sum_{Q}^{Q^{\prime}} v_{q} \psi(x / q)$, where $Q \leq Q^{\prime} \leq 2 Q$ and $\exp \left(B^{\prime} t^{\frac{2}{3}} u^{\frac{1}{3}+\vartheta}\right) \leq Q \leq Q^{\prime} \leq y$, and it is thus sufficient to show that

$$
\sum_{Q}^{Q^{\prime}} v_{q} \psi\left(\frac{x}{q}\right)=O(1 / t) .
$$

This last estimate will follow from

$$
\sum_{Q}^{Q^{\prime}} q v_{q} \psi\left(\frac{x}{q}\right)=O(Q / t)
$$

which we proceed to reduce in turn to Assertion 1 below.

Assertion 1. Let $z>e^{e}, V:=\log z, Q \leq Q^{\prime} \leq 2 Q$ and

$$
\exp \left(\frac{1}{3} B^{\prime} V^{\frac{2}{3}}(\log V)^{\frac{1}{3}+\vartheta}\right) \leq Q \leq Q^{\prime} \leq z \exp \left(-V^{b}\right) .
$$

Then

$$
\left|\sum_{Q}^{Q^{\prime}} q v_{q} e\left(\frac{z}{q}\right)\right| \ll Q V^{-2} .
$$

Lemma 9. Assertion 1 implies estimate (10).

Proof. Let $\exp \left(B^{\prime} t^{\frac{2}{3}} u^{\frac{1}{3}+\vartheta}\right) \leq Q \leq Q^{\prime} \leq x \exp \left(-t^{b}\right)$, and put $R=Q^{\frac{3}{B^{\prime}} t^{1 / 3} u^{-1 / 3-\vartheta}} x^{-1}$ and $z=n x$ for $n \leq R$. For $Y \geq 3$ we have

$$
\begin{aligned}
Y \int_{0}^{\frac{1}{Y}} \psi(y+\theta) d \theta & =-\frac{Y}{2 \pi i} \sum_{n=1}^{\infty} \frac{1}{n} \int_{0}^{\frac{1}{Y}}(e(n(y+\theta))-e(-n(y+\theta))) d \theta \\
& =\sum_{a=-\infty}^{\infty} w_{a} e(a y),
\end{aligned}
$$


where $w_{a}=w_{a, Y}$ satisfies

$$
w_{0}=0 ; \quad w_{a}=-\frac{Y}{2 \pi i a} \int_{0}^{\frac{1}{Y}} e(a \theta) d \theta \quad(a \neq 0)
$$

and

$$
\left|w_{a}\right| \leq \min \left(\frac{1}{|a|}, \frac{Y}{a^{2}}\right) \quad(a \neq 0) .
$$

Thus we have, with $Y=x$,

$$
\begin{aligned}
\left|x \sum_{q=Q}^{Q^{\prime}} q v_{q} \int_{0}^{\frac{1}{x}} \psi\left(\frac{x}{q}+\theta\right) d \theta\right| & =\left|\sum_{q=Q}^{Q^{\prime}} q v_{q} \sum_{a=-\infty}^{\infty} w_{a} e(a x / q)\right| \\
\leq 2 \sum_{n=1}^{\infty}\left|\sum_{q=Q}^{Q^{\prime}} q v_{q} e(n x / q)\right| \min \left(\frac{1}{n}, \frac{x}{n^{2}}\right) & =2 \sum_{n \leq R}+2 \sum_{n>R}=: 2 S_{1}+2 S_{2} .
\end{aligned}
$$

In $S_{1} Q$ and $Q^{\prime}$ satisfy the conditions of Assertion 1 . Indeed note that we have $z \geq x$, whence $V^{-1 / 3}(\log V)^{1 / 3+\vartheta} \leq t^{-1 / 3} u^{1 / 3+\vartheta}$, and $z \leq R x=$ $Q^{\frac{3}{B^{\prime}} t^{1 / 3} u^{-1 / 3-\vartheta}}$. Thus

$$
\exp \left(\frac{1}{3} B^{\prime} V^{\frac{2}{3}}(\log V)^{\frac{1}{3}+\vartheta}\right) \leq \exp \left(\frac{1}{3} B^{\prime} V t^{-\frac{1}{3}} u^{\frac{1}{3}+\vartheta}\right)=z^{\frac{1}{3} B^{\prime} t^{-\frac{1}{3}} u^{\frac{1}{3}+\vartheta}} \leq Q .
$$

Thus we have

$$
\begin{aligned}
S_{1} & =\sum_{n \leq R}\left|\sum_{q=Q}^{Q^{\prime}} q v_{q} e(z / q)\right| \min \left(n^{-1}, x n^{-2}\right) \\
& \ll Q \sum_{n \leq R} V^{-2} \min \left(n^{-1}, x n^{-2}\right) \ll Q t^{-2}\left(\sum_{n \leq x} n^{-1}+x \sum_{n>x} n^{-2}\right) \ll Q t^{-1}
\end{aligned}
$$

As for $S_{2}$ we have, since $R \geq x^{2}$,

$$
S_{2} \ll Q \sum_{n>R} \min \left(n^{-1}, x n^{-2}\right) \ll Q x \sum_{n>x^{2}} n^{-2} \ll Q x^{-1} .
$$

We just proved that

$$
\left|x \sum_{q=Q}^{Q^{\prime}} q v_{q} \int_{0}^{\frac{1}{x}} \psi\left(\frac{x}{q}+\theta\right) d \theta\right| \ll Q t^{-1}
$$

Now

$$
\psi\left(\frac{x}{q}+\theta\right)-\psi\left(\frac{x}{q}\right)=\theta-\left(\left[\frac{x}{q}+\theta\right]-\left[\frac{x}{q}\right]\right)=\theta-\sum_{\frac{x}{q}<m<\frac{x}{q}+\theta} 1
$$


and thus

$$
x \int_{0}^{\frac{1}{x}} \psi\left(\frac{x}{q}+\theta\right) d \theta-\psi\left(\frac{x}{q}\right)=\frac{1}{2 x}+O\left(\sum_{\frac{x}{q}<m<\frac{x}{q}+\frac{1}{x}} 1\right)
$$

and with (h1)

$$
\begin{aligned}
\left|x \sum_{q=Q}^{Q^{\prime}} q v_{q} \int_{0}^{\frac{1}{x}} \psi\left(\frac{x}{q}+\theta\right) d \theta-\sum_{Q}^{Q^{\prime}} q v_{q} \psi\left(\frac{x}{q}\right)\right| \ll Q x^{-1}(\log Q)^{\alpha} & +\sum_{x<m q \leq x+2 Q / x}\left|q v_{q}\right| .
\end{aligned}
$$

This completes the proof of the lemma, since $2 Q<x$ and the last sum is empty.

Now we reduce Assertion 1 to Assertion 2.

Assertion 2. If $\exp \left(\frac{1}{3} B^{\prime} t^{2 / 3} u^{1 / 3+\vartheta}\right) \leq N \leq x \exp \left(-t^{b}\right)$ then

$$
\left|\sum_{q \leq N} q v_{q} e\left(\frac{x}{q}\right)\right| \ll N s^{-4}
$$

Lemma 10. Assertion 2 implies Assertion 1.

Proof. We apply twice Assertion 2: with $x=z, N=Q$, and with $x=z$, $N=Q^{\prime}$. We obtain

$$
\left|\sum_{Q}^{Q^{\prime}} q v_{q} e\left(\frac{x}{q}\right)\right| \leq\left|\sum_{q \leq Q^{\prime}} q v_{q} e\left(\frac{x}{q}\right)\right|+\left|\sum_{q \leq Q} q v_{q} e\left(\frac{x}{q}\right)\right| \ll Q(\log Q)^{-4} \ll Q V^{-2},
$$

so that Assertion 1 is satisfied and the lemma is proved.

We reduce Assertion 2 to Assertion 3.

Assertion 3. If

$$
S_{1}(\nu, U):=\sum_{U \leq p^{\nu} \leq U^{\prime}} p^{\nu} v\left(p^{\nu}\right) \sum_{q \leq N_{0} p^{-\nu}} q v_{q} e\left(\frac{x}{p^{\nu} q}\right)
$$

and

$$
S_{2}(\nu, U):=\sum_{U \leq p^{\nu} \leq U^{\prime}} p^{\nu} v\left(p^{\nu}\right) \sum_{\substack{N_{0}<q \leq N p^{-\nu} \\ g(q)<p}} q v_{q} e\left(\frac{x}{p^{\nu} q}\right)
$$

where $N_{0}^{\nu} \leq U \leq U^{\prime} \leq 2 U \leq 2 N N_{0}^{-1}$ and where $g(q)$ denotes the largest prime divisor of $q$, then

$$
\left.\begin{array}{ll}
\text { if } \vartheta=1 & \left|S_{2}(\nu, U)\right| \\
\text { if } \vartheta=2 & \left|S_{1}(\nu, U)\right|+\left|S_{2}(\nu, U)\right|
\end{array}\right\} \ll N s^{-6}
$$


where the implied constants are independant of $\nu$.

Lemma 11. Assertion 3 implies Assertion 2.

Proof. We write

$$
\sum_{q \leq N} q v_{q} e\left(\frac{x}{q}\right)=\sum_{\substack{q \leq N \\ g(q)>N_{0}}} q v_{q} e\left(\frac{x}{q}\right)+\sum_{\substack{q \leq N \\ g(q) \leq N_{0}}} q v_{q} e\left(\frac{x}{q}\right)=: S_{3}+S^{\prime}
$$

and we first estimate $\left|S^{\prime}\right|$. We have

$$
\left|S^{\prime}\right| \ll \sum_{q \leq N^{1 / 2}} q\left|v_{q}\right|+\sum_{\substack{N^{1 / 2} \leq q \leq N \\ g(q) \leq N_{0}}} q\left|v_{q}\right|=: I+I I .
$$

We have from (h1)

$$
I=O\left(N^{1 / 2} \log ^{\alpha} N\right) .
$$

As for $I I$, suppose first that the hypotheses of Theorem 1 hold. We have

$$
I I^{2} \leq \Psi\left(N, N_{0}\right) \sum_{n \leq N}\left(n v_{n}\right)^{2},
$$

where $\Psi\left(N, N_{0}\right)$ denotes the number of integers not exceeding $N$ and free of prime factors larger than $N_{0}$. Since

$$
\Psi\left(N, N_{0}\right) \ll N^{1-\frac{1}{2 \log N_{0}}}=N s^{-\frac{k_{0}}{2}}
$$

(see for instance Theorem III.5.1 in [8]), and since $k_{0} \geq 16+2 \beta$ we have from (h2)

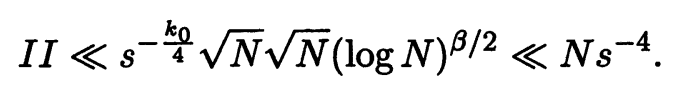

Hence in this case

$$
\left|S^{\prime}\right| \ll N s^{-4}
$$

Now assume instead that (h4) of Theorem 2 holds. Then, since $g(q) \leq N_{0}$ we have $N_{0}^{\Omega(q)} \geq N^{1 / 2}$, whence $\Omega(q) \geq \frac{k_{0}}{2} \log s$ and

$$
\gamma^{\Omega(q)} \geq s^{\frac{k_{0} \log \gamma}{2}}
$$

From (h4) it follows that

$$
I I \ll s^{-\frac{k_{0} \log \gamma}{2}} \sum_{q \leq N} q\left|v_{q}\right| \gamma^{\Omega(q)} \ll N s^{a-\frac{k_{0} \log \gamma}{2}} .
$$

And since $k_{0} \geq(a+4) 2 / \log \gamma$ we also have in this case

$$
\left|S^{\prime}\right| \ll N s^{-4} \text {. }
$$

There remains to estimate $S_{3}$. 
We write

$$
S_{3}=\sum_{\nu=1}^{[s]} S_{3}(\nu) \quad \text { and } \quad S_{3}(\nu)=S_{1}(\nu)+S_{2}(\nu)
$$

where

$$
S_{1}(\nu):=\sum_{q \leq N_{0}} q v_{q} \sum_{N_{0}<p \leq\left(N q^{-1}\right)^{1 / \nu}} p^{\nu} v\left(p^{\nu}\right) e\left(\frac{x}{p^{\nu} q}\right)
$$

and

$$
S_{2}(\nu):=\sum_{N_{0}^{\nu} \leq p^{\nu} \leq N N_{0}^{-1}} p^{\nu} v\left(p^{\nu}\right) \sum_{\substack{N_{0}<q \leq N p^{-\nu} \\ g(q)<p}} q v_{q} e\left(\frac{x}{p^{\nu} q}\right)
$$

First we assume that the hypotheses of Theorem 1 hold, and we evaluate $S_{1}(\nu)$. Its inner sum is, if we let $p_{n}$ denote the $n$-th prime number,

$$
\sum_{N_{0}<p_{n} \leq\left(N q^{-1}\right)^{1 / \nu}} W\left(p_{n}\right)\left(z\left(p_{n}\right)-z\left(p_{n+1}\right)\right)+W\left(p_{\lambda}\right) z\left(p_{\lambda+1}\right),
$$

where

$$
W\left(p_{n}\right):=\sum_{N_{0}<p \leq p_{n}} e\left(\frac{x}{p^{\nu} q}\right), \lambda:=\pi\left(\left(N q^{-1}\right)^{1 / \nu}\right)
$$

and

$$
z\left(p_{n}\right)=z\left(p_{n}, \nu\right):=p_{n}^{\nu} v\left(p_{n}^{\nu}\right)
$$

If $\nu \geq 3$ we are content with the estimate $\left|W\left(p_{n}\right)\right| \ll N^{1 / \nu}$. And if $\nu=1$ or 2 we have $\left|W\left(p_{n}\right)\right| \leq N^{3 /(4 \nu)}$ when $p_{n} \leq N^{3 /(4 \nu)}$, and

$$
W\left(p_{n}\right)=-\sum_{p<N_{0}} e\left(\frac{x}{p^{\nu} q}\right)+\sum_{p<p_{n}} e\left(\frac{x}{p^{\nu} q}\right)=O\left(\left(N q^{-1}\right)^{\frac{1}{\nu}} s^{-\alpha-5}\right)
$$

when $p_{n}>N^{3 /(4 \nu)}$, since then $p_{n}^{\nu} \geq \exp \left(\frac{3}{4} \frac{1}{3} 4 B t^{2 / 3} u^{4 / 3}\right)$ (recall that $B^{\prime} \geq$ $4 B$ ), and the Main Lemma applies with $\Lambda=5+\alpha, x / q$ instead of $x$ and $p_{n}^{\nu}$ instead of $N$. Hence, with (h3) we see that the expression in (11) above is $O$ of

$$
\begin{array}{r}
\left(N q^{-1}\right)^{\frac{1}{\nu}} s^{-5-\alpha}\left(\sum_{N_{0}<p_{n} \leq\left(N q^{-1}\right)^{1 / \nu}}\left|z\left(p_{n}\right)-z\left(p_{n+1}\right)\right|+\left|z\left(p_{\lambda+1}\right)\right|\right) \\
\ll N q^{-1} s^{-5-\alpha} \quad \text { if } \nu=1 \text { or } 2
\end{array}
$$

and of

$$
N^{1 / \nu} \sum_{p \leq N^{1 / \nu}} p^{\nu}\left|v\left(p^{\nu}\right)\right| \ll N^{\frac{2}{3}} \quad \text { if } \nu>2
$$


the implied constant being independent of $\nu$ in the second estimate. With (h1) it follows that

$$
\sum_{\nu=3}^{[s]}\left|S_{1}(\nu)\right| \ll N^{\frac{2}{3}} N_{0} s^{\alpha+1} \ll N^{\frac{3}{4}},
$$

and with Remark 1 that, for $\nu=1$ and $\nu=2$,

$$
\left|S_{1}(\nu)\right|=\sum_{q \leq N_{0}} q\left|v_{q}\right| q^{-1} N s^{-5-\alpha} \ll N s^{-4} .
$$

Finally, with Assertion 3 we see that

$$
\left|S_{2}(\nu)\right| \ll N s^{-5}
$$

for every $\nu$ (and for an implied absolute constant), and the lemma is proved in this case.

Now we assume that the hypotheses of Theorem 2 hold. First we evaluate $S_{3}(\nu)=S_{1}(\nu)+S_{2}(\nu)$ when $\nu \leq K_{0}$. The inner sum of $S_{1}(\nu)$ is as in (11). Now we may rewrite $S_{2}(\nu)$ as

$$
S_{2}(\nu):=\sum_{N_{0}<q \leq N N_{0}^{-1}} q v_{q} \sum_{N_{0}^{\prime}<p \leq\left(N q^{-1}\right)^{1 / \nu}} p^{\nu} v\left(p^{\nu}\right) e\left(\frac{x}{p^{\nu} q}\right)
$$

where $N_{0}^{\prime}=N_{0}^{\prime}(q):=\max \left\{N_{0}, g(q)\right\}$, and its inner sum as

$$
\sum_{N_{0}^{\prime}<p_{n} \leq\left(N q^{-1}\right)^{1 / \nu}} W\left(p_{n}\right)\left(z\left(p_{n}\right)-z\left(p_{n+1}\right)\right)+W\left(p_{\lambda}\right) z\left(p_{\lambda+1}\right),
$$

As before we have

$$
W\left(p_{n}\right)=-\sum_{p<N_{0}} e\left(\frac{x}{p^{\nu} q}\right)+\sum_{p<p_{n}} e\left(\frac{x}{p^{\nu} q}\right) .
$$

This time, since

$$
p_{n}^{\nu} \geq N_{0}^{\nu}=\exp \left(\frac{\nu s}{k_{0} \log s}\right) \geq \exp \left(\frac{k_{0} B t^{2 / 3} u^{7 / 3}}{k_{0} u}\right)=\exp \left(B t^{2 / 3} u^{4 / 3}\right)
$$

(recall that $B^{\prime} \geq 3 k_{0} B$ ), and since $p_{n}^{\nu} \leq N / q$, the Main Lemma of Section 2 applies to both sums, with $\Lambda=\alpha+5, x / q$ instead of $x$ and $p_{n}^{\nu}$ or $N_{0}^{\nu}$ instead of $N$. So for $\nu \leq K_{0}$ we have

$$
\left|W\left(p_{n}\right)\right| \ll p_{n} s^{-\alpha-5} \ll N^{\frac{1}{\nu}} q^{-\frac{1}{\nu}} s^{-\alpha-5} .
$$

Hence we see with $\left(\mathrm{h} 3^{*}\right)$ that the expressions in (11) and (12) above are both $O$ of

$$
N^{\frac{1}{\nu}} q^{-\frac{1}{\nu}} s^{-5-\alpha}\left(\sum_{N_{0}<p_{n} \leq\left(N q^{-1}\right)^{\frac{1}{\nu}}}\left|z\left(p_{n}\right)-z\left(p_{n+1}\right)\right|+\left|z\left(p_{\lambda+1}\right)\right|\right) \ll N q^{-1} s^{-5-\alpha}
$$


if $\nu \leq K_{0}$, whence with Remark 1 ,

$$
\left|S_{1}(\nu)\right|+\left|S_{2}(\nu)\right| \ll N \sum_{q \leq N} q\left|v_{q}\right| q^{-1} s^{-5-\alpha} \ll N s^{-4} .
$$

Finally we see by Assertion 3 that

$$
\sum_{K_{0}<\nu \leq[s]}\left(\left|S_{1}(\nu)\right|+\left|S_{2}(\nu)\right|\right) \ll N s^{-4}
$$

whence Assertion 2 holds for $\vartheta=2$ also: the lemma is proved.

Now we use Lemmata 6 and 7 of Section 2 to prove that Assertion 3 holds, thus concluding the proof of the theorems. Let $T(v, 1, U)$ be as defined just before Lemma 4 (with $M=1$ and $k=1$ ), and $\tau(v, U)$ be as in Lemma 7.

Lemma 12. There are some $V, V^{\prime}$ satisfying

$$
U \leq V=V\left(q, q_{1}\right) \leq V^{\prime}=V^{\prime}\left(q, q_{1}\right) \leq 2 U \text {. }
$$

such that

$$
\begin{array}{ll}
\text { if } \vartheta=1 \text { and } \nu \geq 1, \text { or } \\
\text { if } \vartheta=2 \text { and } \nu>K_{0} \text { then } & \left|S_{2}(\nu, U)\right|^{2} \ll T(v, 1, U)+N^{2} s^{-\frac{k_{2}}{2}+2 \alpha} ; \\
\text { if } \vartheta=2 \text { and } \nu>K_{0} \text { then } & \left|S_{1}(\nu, U)\right|^{2} \ll \tau(v, U)+N^{2} s^{-\frac{k_{2}}{2}+2 \alpha} ;
\end{array}
$$

where the implied constants are independent of $\nu$

Proof. We estimate $\left|S_{2}(\nu, U)\right|^{2}$. The estimation of $\left|S_{1}(\nu, U)\right|^{2}$ is done very similarly (and in a simpler way). We have by the Cauchy-Schwarz inequality, and by (h3) if $\vartheta=1$ and $\left(\mathrm{h} 2^{*}\right)$ if $\vartheta=2$,

$$
\begin{aligned}
\left|S_{2}(\nu, U)\right|^{2} & \leq \sum_{U \leq p^{\nu} \leq U^{\prime}}\left(p^{\nu} v\left(p^{\nu}\right)\right)^{2} \sum_{U \leq p^{\nu} \leq U^{\prime}}\left|\sum_{\substack{N_{0}<q \leq N p^{-\nu} \\
g(q)<p}} q v_{q} e\left(\frac{x}{p^{\nu} q}\right)\right|^{2} \\
& \ll U \sum_{U \leq n \leq U^{\prime}}\left|\sum_{\substack{N_{0}<q \leq N n^{-1} \\
g(q)<n^{1 / \nu}}} q v_{q} e\left(\frac{x}{n q}\right)\right|^{2}
\end{aligned}
$$

If we set $V:=\max \left(U, g(q)^{\nu}+1, g\left(q_{1}\right)^{\nu}+1\right)$ and $V^{\prime}:=\min \left(U^{\prime},\left[N q^{-1}\right],\left[N q_{1}^{-1}\right]\right)$, we have

$$
\left|S_{2}(\nu, U)\right|^{2} \ll U \sum_{q, q_{1} \leq N U^{-1}}\left|q v(q) q_{1} v\left(q_{1}\right)\right|\left|\sum_{V}^{V^{\prime}} e\left(\frac{x z}{n}\right)\right|,
$$

where $z$ denotes as before $q_{1}^{-1}-q^{-1}$. With the help of (h2) if $\vartheta=1$ and $\left(\mathrm{h} 2^{*}\right)$ if $\vartheta=2$ the contribution from the terms with $q=q_{1}$ on the right of this last estimate is easily seen to contribute a $O\left(N^{2} s^{-k_{2}}\right)$. And it is 
clearly sufficient to estimate only half the remaining terms in (13), i.e. the terms with $q_{1}<q$. Now the conditions

$$
\left\{\begin{array}{c}
N U^{-1} s^{-k_{2}} \leq q_{1}<q \leq N U^{-1} \\
q-q_{1}>N U^{-1} s^{-k_{2}}
\end{array}\right\}
$$

imply

$$
\left\{\begin{array}{c}
N U^{-1} s^{-k_{2}} \leq q_{1}<q \leq N U^{-1} \\
N^{-1} U s^{-k_{2}} \leq z \leq N^{-1} U s^{k_{2}}
\end{array}\right\} .
$$

We estimate the terms moved aside to replace the condition $q_{1}<q \leq$ $N U^{-1}$ by (14). First by using (h1) we easily see that if we restrict the sum to the terms with $N U^{-1} s^{-k_{2} / 2} \leq q_{1}<q \leq N U^{-1}$, the terms moved aside contribute at most $O\left(N^{2} s^{-k_{2} / 2+2 \alpha}\right)$. So we may suppose that $q_{1} \geq$ $N U^{-1} s^{-k_{2} / 2}$, that is $N U^{-1} s^{-k_{2}} \leq q_{1} s^{-k_{2} / 2}$, whence with Lemma 8

$$
\sum_{q_{1}<q<q_{1}+N U^{-1} s^{-k_{2}}}|q v(q)| \ll q_{1}\left(\log q_{1}\right)^{-\frac{k_{2}}{2}+\alpha} \ll N U^{-1} s^{-\frac{k_{2}}{2}+\alpha} .
$$

It follows that the terms moved aside to ensure that $q-q_{1}>N U^{-1} s^{-k_{2}}$ contribute at most a $O\left(N^{2} s^{-k_{2} / 2+2 \alpha}\right)$. On comparing (15) with the definition of $T(v, 1, U)$, we conclude the proof of the required estimate for $\left|S_{2}(k, U)\right|^{2}$.

By Lemma 12, (8) and the choice of $B$ the theorems are proved.

\section{SoME APPLICATIONS}

I first state three applications of Theorem 1.

Theorem 3. Let $\sigma$ denote the usual sum-of-divisors function, $\phi$ the Euler phi function, and $r$ some real number. We have

$$
\begin{aligned}
& \sum_{n \leq x}\left(\frac{\sigma(n)}{n}\right)^{r}=C_{0} x+\sum_{m=0}^{[r]} A_{m}(\log x)^{r-m}-E_{0}(x)+o(1) \\
& \sum_{n \leq x}\left(\frac{\phi(n)}{n}\right)^{r}=C_{1} x+\sum_{m=0}^{[r]} B_{m}(\log x)^{r-m}-E_{1}(x)+o(1) \\
& \sum_{n \leq x}\left(\frac{\sigma(n)}{\phi(n)}\right)^{r}=C_{2} x+\sum_{m=0}^{[2 r]} D_{m}(\log x)^{2 r-m}-E_{2}(x)+o(1) .
\end{aligned}
$$

where the $C_{i}, A_{m}, B_{m}$ and $D_{m}$ are real constants, and where

$$
E_{i}(x)=\sum_{n \leq y} v_{i} \psi\left(\frac{x}{n}\right) \quad(i=0,1,2)
$$


with $y$ and $v=v_{i}$ as in Theorem 1, whence

$$
\begin{gathered}
E_{i}=O\left((\log x)^{\frac{2|r|}{3}}(\log \log x)^{\frac{4|r|}{3}}\right)(i=0,1) \quad \text { and } \\
E_{2}=O\left((\log x)^{\frac{4|r|}{3}}(\log \log x)^{\frac{8|r|}{3}}\right) .
\end{gathered}
$$

Proof. As it is mentioned in the introduction the proof of (19) is to be found in [1], with the restriction that only a weaker version of (h1), not sufficient to ensure the validity of $(20)$, is verified there. (On the other hand a stronger version of (h3), which is not needed anymore, is also obtained there). I briefly indicate how (20) is established for $i=0$ by using the results of [1] and Theorem 1 of this paper (the proofs for $i=1,2$ are similar). For $v=v_{0}$ we have $(\sigma(n) / n)^{r}=(1 * v)(n)$ with

$$
v\left(p^{k}\right)=\left(1+\frac{1}{p}+\cdots+\frac{1}{p^{k}}\right)^{r}-\left(1+\frac{1}{p}+\cdots+\frac{1}{p^{k-1}}\right)^{r}
$$

(Lemma 5.1 of [1]). It follows that $\sum|v(n)| n^{-s}=\zeta^{|r|}(s+1) f(s+1)$, where the function $f(s)$ can be represented by a Dirichlet series whose abscissa of absolute convergence is strictly smaller than 1. Thus Lemma 2.3 of [1] applies to $\sum_{n \leq x} v(n)$ (with $\alpha=|r|$ ), and this immediately implies (h1), with $\alpha=|r|-1$. For a proof of (h2) see Lemma 5.3 of [1]. And with (21) we easily see that $(\mathrm{h} 3)$ is satisfied.

We now turn to an application of Theorem 2. Let $n$ be a positive integer with canonical decomposition $n=p_{1}^{\nu_{1}} \cdots p_{k}^{\nu_{k}}$. We call $d$ an exponential divisor of $n$ if $d=p_{1}^{\mu_{1}} \cdots p_{k}^{\mu_{k}}$ with $\mu_{j} \mid \nu_{j}(1 \leq j \leq k)$. We can consider the sum of all exponential divisors of $n$, which we denote by $\sigma^{(e)}(n)$ (with the convention that $\sigma^{(e)}(1)=1$ ), or a sum $\sigma^{(r)}(n)$ restricted to the exponential divisors of $n$ of a certain type (and also with the convention that $\sigma^{(r)}(1)=$ 1). For instance if we exclude in $\sigma^{(r)}(n)$ the divisors of $n$ for which $\mu_{j}=\nu_{j} / 2$ when $\nu_{j}>2$, then we have $\sigma^{(r)}(n) / n=\left(1 * v_{r}\right)(n)$, where for $v=v_{r}$

$$
v\left(p^{\alpha}\right)=\sum_{\substack{d \mid \alpha, d \neq \alpha / 2 \text { if } \alpha>2}} \frac{1}{p^{\alpha-d}}-\sum_{\substack{d \mid \alpha-1, d \neq(\alpha-1) / 2 \text { if } \alpha>3}} \frac{1}{p^{\alpha-1-d}}
$$

It follows that, for some $H_{0}(s)$ represented by a Dirichlet series with abscissa of absolute convergence $\sigma_{a}\left(H_{0}\right)<0$, we have

$$
\begin{aligned}
\sum_{n \geq 0} \frac{v(n)}{n^{s}} & =\prod_{p}\left(1+\frac{\frac{1}{p}}{p^{2 s}}+\frac{\frac{1}{p^{2}}-\frac{1}{p}}{p^{3 s}}+\frac{\frac{1}{p^{3}}-\frac{1}{p^{2}}}{p^{4 s}}+\frac{\frac{1}{p^{4}}-\frac{1}{p^{3}}}{p^{5 s}}+\frac{\frac{1}{p^{5}}}{p^{6 s}}+\cdots\right) \\
& =\frac{\zeta(2 s+1)}{\zeta(3 s+1)} H_{0}(s)
\end{aligned}
$$


and

$$
\sum_{n \geq 0} \frac{\sigma^{(r)}(n) / n}{n^{s}}=\zeta(s) \frac{\zeta(2 s+1)}{\zeta(3 s+1)} H_{0}(s) .
$$

Similarly we see that

$$
\begin{aligned}
\sum_{n \geq 0} \frac{|v(n)|}{n^{s}} & =\zeta(2 s+1) \zeta(3 s+1) H_{1}(s) \quad\left(\sigma_{a}\left(H_{1}\right)<0\right) \quad \text { and } \\
\sum_{n \geq 0} \frac{(n v(n))^{2}}{n^{s}} & =\zeta(3 s-4) H_{2}(s) \quad\left(\sigma_{a}\left(H_{2}\right)<\frac{5}{3}\right) .
\end{aligned}
$$

Now if we use well-known estimates for $\zeta(2 s+1)$ and $\zeta(3 s+1)$ in a large enough zero-free region of the function $\zeta$ (see for instance Chapitre II.3 of [8]), a standard complex integration argument invoking Perron's inversion formula and the theorem of residues, exactly as in a classical proof of the prime number theorem (see for instance Section II.4 and the introductory paragraph of Section II.5 in [8]) yields

$$
\begin{aligned}
\sum_{n \leq x}|v(n)| & =C_{0} \log ^{2} x+C_{1} \log x+C_{2}+O\left(\exp \left(-C_{3} \sqrt{\log x}\right)\right) \\
\sum_{n \leq x}(n v(n))^{2} & =O\left(x^{5 / 3}\right) .
\end{aligned}
$$

Note that of course a more precise estimate can be obtained instead of (24), which is however sufficient to ensure that the first part of $(\mathrm{h} 2 *)$ is satisfied. And by (23) hypothesis (h1) (with $\alpha=1$ ) is also satisfied. Now for $1<\gamma<2^{1 / 4} \leq p^{1 / 4}$ and $k \geq 44$ we have, for $0<c_{k} \leq \gamma^{k} k p^{(-k-1) / 2}<$ $k p^{-k / 4}<p^{-k / 8}$,

$$
\begin{aligned}
& \sum_{n \geq 0} \frac{\gamma^{\Omega(n)}|v(n)|}{n^{s}}=\prod_{p}\left(1+\frac{\gamma^{2}\left(\frac{1}{p}\right)}{p^{2 s}}+\frac{\gamma^{3}\left(\frac{1}{p}-\frac{1}{p^{2}}\right)}{p^{3 s}}+\cdots+\frac{c_{k}}{p^{k s}}+\cdots\right) \\
= & \zeta^{\gamma^{2}}(2 s+1) \zeta^{\gamma^{3}}(3 s+1) \zeta^{\gamma^{4}}(4 s+2) H_{3}^{\prime}(s) \prod_{p}\left(1+O\left(\frac{1}{p^{k(s+1 / 8)}}\right)\right) \\
= & \zeta^{\gamma^{2}}(2 s+1) \zeta^{\gamma^{3}}(3 s+1) H_{3}(s) \quad\left(\sigma_{a}\left(H_{3}^{\prime}\right)<0 \text { and } \sigma_{a}\left(H_{3}\right)<0\right) \\
= & \sum_{n \geq 0} \frac{a(n)}{n^{s}} \sum_{n \geq 0} \frac{b(n)}{n^{s}} \sum_{n \geq 0} \frac{c(n)}{n^{s}}
\end{aligned}
$$

We clearly have $\sum_{n \leq x}|c(n)|=O(1)$, and since

$$
\zeta^{\gamma^{2}}(2 s+1)=\prod\left(1-\frac{1}{p^{2 s+1}}\right)^{-\gamma^{2}}=\prod\left(1+\frac{\left(\begin{array}{c}
\gamma^{2} \\
1
\end{array}\right)}{p^{2 s+1}}+\frac{\left(\begin{array}{c}
\gamma^{2} \\
2
\end{array}\right)}{p^{4 s+2}}+\cdots\right),
$$


we have $a(n)=0$ if $n$ is not squareful, and

$$
|a(n)|=\frac{1}{n^{1 / 2}}\left|\left(\begin{array}{c}
\gamma^{2} \\
\alpha_{1}
\end{array}\right) \cdots\left(\begin{array}{c}
\gamma^{2} \\
\alpha_{k}
\end{array}\right)\right|<\frac{\gamma^{2 \omega(n)}}{n^{1 / 2}}<\frac{2^{\omega(n)}}{n^{1 / 2}}
$$

if $n=p_{1}^{\alpha_{1}} \cdots p_{k}^{\alpha_{k}}$. Similarly $b(n)=0$ if $n$ is not cubeful, and $|b(n)|<$ $2^{\omega(n)} n^{-1 / 3}$. It follows that

$$
\begin{aligned}
\sum_{n \leq x} \gamma^{\Omega(n)}|v(n)| & =\sum_{n \leq x}(a * b * c)(n) \\
& =\sum_{d \leq x} c(d) \sum_{m=n_{1}^{2} n_{2}^{3} \leq x / d} a\left(n_{1}^{2}\right) b\left(n_{2}^{3}\right) \\
& \leq \sum_{d \leq x}|c(d)| \sum_{n_{1} \leq(x / d)^{1 / 2}} \frac{2^{\omega\left(n_{1}\right)}}{n_{1}} \sum_{n_{2} \leq\left(x / d n_{1}^{2}\right)^{1 / 3}} \frac{2^{\omega\left(n_{2}\right)}}{n_{2}} \\
& =O\left((\log x)^{4}\right),
\end{aligned}
$$

where we have used E. Grosswald's estimate $\sum_{n \leq N} 2^{\omega(n)}=O(N \log N)$ (see [3]). Note that by using an argument similar to that required to obtain Theorem 3 (see Chapitre II.5 of [8] or [1]) one can show that in fact

$$
\sum_{n \leq x} \gamma^{\Omega(n)}|v(n)| \sim c_{\gamma}(\log x)^{\gamma^{2}+\gamma^{3}}
$$

But (25) is sufficient for our purpose, which is to ensure that (h4) holds. Moreover from (22) it is easy to verify that the second part of (h2*), as well as $(\mathrm{h} 3 *)$, is satisfied for $\nu>K_{0}:=3$. Now if we note that

$$
\sum_{n \leq x} \frac{\sigma^{(r)}(n)}{n}=x \sum_{n \leq x} \frac{v(n)}{n}-\frac{1}{2} \sum_{n \leq x} v(n)-\sum_{n \leq x} v(n) \psi\left(\frac{x}{n}\right)
$$

that, again with a standard complex integration argument we have

$$
\begin{aligned}
& \sum_{n \leq x} v(n)=D_{0}+O\left(\exp \left(-D_{1} \sqrt{\log x}\right)\right)=: D_{0}+R(x) \text { and } \\
& \sum_{n \leq x} \frac{v(n)}{n}=D_{2}+O(1 / x)
\end{aligned}
$$

where the $D_{i}$ are some effective constants, and finally that for $y$ as in the statement of Theorem 2 we have

$$
\sum_{y<n \leq x} v(n) \psi\left(\frac{x}{n}\right)=\int_{y}^{x} \psi\left(\frac{x}{t}\right) d R(t)=O\left(\exp \left(-D_{1} \sqrt{\log x}\right)\right)
$$

we see that all the assumptions of Theorem 2 are satisfied by $v=v_{r}$. Thus we may state 
Theorem 4. We have

$$
\sum_{n \leq x} \frac{\sigma^{(r)}(n)}{n}=C_{3} x-E_{3}(x)+O(1)
$$

where

$$
E_{3}(x)=\sum_{n \leq y} v_{r} \psi\left(\frac{x}{n}\right)=O\left((\log x)^{\frac{4}{3}}(\log \log x)^{\frac{14}{3}}\right) .
$$

Remark 6. In [6] we establish Theorem 4A. We have

$$
\sum_{n \leq x} \frac{\sigma^{(e)}(n)}{n}=C_{4} x-E_{4}(x)+O(1)
$$

where

$$
E_{4}(x)=\sum_{n \leq y} v_{e} \psi\left(\frac{x}{n}\right)=O\left((\log x)^{\frac{5}{3}}\right) .
$$

With practically no modification the method we use there (see "Proof of Theorem 2" in [6]) yields

$$
E_{3}(x)=O\left((\log x)^{\frac{5}{3}}\right),
$$

which of course is not quite as good as Theorem 4 just above. On the other hand, however, we cannot improve Theorem $4 A$ with the help of Theorem 2, which is not applicable in this case: the function $v_{e}$ doesn't satisfy the second part of (h2*).

\section{REMARKS ON SALTYKOV'S PAPER 7}

Saltykov proves in [7] (Lemma 1.5) that if, under certain conditions on the coefficients of the polynomial $f(y)=\alpha_{n+1} y^{n+1}+\cdots+\alpha_{1} y$, we have

$$
\left|\sum_{y=1}^{P} e(f(y))\right| \leq \exp \left(c_{1} n^{\gamma_{1}}\right) P^{1-\frac{c_{2}}{n^{\gamma}}}
$$

for some positive absolute constants $c_{1}$ and $c_{2}$, where $\gamma_{1} \geq 0, \gamma_{2} \geq 1$ and $1+\gamma_{2}>\gamma_{1}$, then, if $A>0$ is a sufficiently large constant depending upon $\gamma_{1}$ and $\gamma_{2}$, and $\log z>(12 A)^{\gamma_{1}+\gamma_{2}+1}$, if $\beta$ belongs to the interval $\left[A(\log z)^{1 /\left(\gamma_{1}+\gamma_{2}+1\right)}, 1 / 12\right]$, and if $Q$ and $Q^{\prime}$ are integers such that $Q<Q^{\prime} \leq$ $2 Q$ and $Q=\left[z^{\beta}\right]$, we have

$$
\left|\sum_{y=Q}^{Q^{\prime}} e(z / y)\right| \ll Q \exp \left(-c_{3} \beta^{1+\gamma_{2}} \log z+c_{4} \beta^{-\gamma_{1}}\right),
$$

where $c_{3}$ and $c_{4}$ are some positive constants (depending on $\gamma_{1}$ and $\gamma_{2}$ ). 
In the proof of Lemma 2.6 he then applies this result, obtaining

$$
\sum_{n=V}^{V^{\prime}} e(x w / n) \ll M U \exp \left(-c_{3} \beta^{1+\gamma_{2}} \log (x w)+c_{4} \beta^{-\gamma_{1}}\right)
$$

where $M U \leq V \leq V^{\prime} \leq 2 M U$ and

$$
w=N^{-1} H^{\lambda} M U \text { with }
$$

$\exp \left(B t^{\gamma} u^{1+\epsilon}\right) \leq N \leq x \exp \left(-t^{\frac{1}{2}}\right), \quad \gamma:=\frac{\gamma_{1}+\gamma_{2}}{\gamma_{1}+\gamma_{2}+1}, \quad t:=\log x, \quad u:=\log t$, $H:=(\log N)^{33}=: s^{33}$, and where $\beta=\beta(\lambda)$ is defined by

$$
M U=(x w)^{\beta}=\left(x N^{-1} H^{\lambda} M U\right)^{\beta} \quad\left(\text { whence } M U=\left(x N^{-1} H^{\lambda}\right)^{\frac{\beta}{1-\beta}}\right. \text { ) }
$$

and belongs to the interval

$$
\left[A\left(\log \left(x N^{-1} H^{\lambda}\right)\right)^{-\frac{1}{\gamma_{1}+\gamma_{2}+1}}, \frac{1}{12}\right] .
$$

Saltykov claims that

$$
\left|\sum_{n=V}^{V^{\prime}} e(x w / n)\right| \ll M U \exp \left(-c_{3} \beta^{1+\gamma_{2}} \log (x w)+c_{4} \beta^{-\gamma_{1}}\right)=O\left(M U H^{-1}\right)
$$

(first displayed formula after (37) on page 43).

This estimate is essential for the proof of $(\mathrm{S})$ in the introduction, with $\gamma=2 / 3$. But the only values of $\gamma_{1} \geq 0$ and $\gamma_{2} \geq 1$ for which $\gamma=2 / 3$ and for which estimate (26) is proved are $\gamma_{1}=0$ and $\gamma_{2}=2$. No stronger estimate is given in Theorem 1 of Korobov's [5], used by Saltykov (nor in Theorem 1 of Karatsuba's [4], used in the present paper). And for these values of $\gamma_{1}$ and $\gamma_{2}$ the equality in (30) is not satisfied when $\beta=A\left(\log \left(x N^{-1} H^{\lambda}\right)\right)^{-1 / 3}$. Indeed, since

$$
\log (M U)=\frac{\beta}{1-\beta} \log \left(x N^{-1} H^{\lambda}\right) \ll 2 \beta \log \left(x N^{-1} H^{\lambda}\right),
$$

the middle expression in $(30)$ is then

$$
\begin{aligned}
& M U \exp \left(-c_{3} \beta^{3} \log (x w)+c_{4} \beta^{0}\right) \\
\gg & M U \exp \left(-c_{3} \beta^{3} \log (x w)\right)=M U \exp \left(-c_{3} \beta^{2} \log (M U)\right) \\
\gg & M U \exp \left(-2 c_{3} \beta^{3} \log \left(x N^{-1} H^{\lambda}\right)\right)=M U \exp \left(-2 c_{3} A^{3}\right) \\
\gg & M U .
\end{aligned}
$$

In fact, Saltykov's argument is correct as soon as $\gamma_{1}>0$, and thus yields (with $\gamma_{2}=2$ )

$$
H(x)=O\left((\log x)^{\frac{2}{3}+\epsilon}\right)
$$


for every $\epsilon>0$, which is the estimate he claims to improve upon (and which he attributes to Korobov: see below (9) on page 35 of [7]). If however, similarly as in Lemma 4 in Section 2 above, we make the stronger requirement that $\beta$ be in the subinterval of (29)

$$
\left[A\left(\log \left(x N^{-1} H^{\lambda}\right)\right)^{-\frac{1}{3}} u^{\frac{1}{3}}, \frac{1}{12}\right]
$$

then we have instead

$$
M U \exp \left(-c_{3} \beta^{3} \log (x w)\right) \ll M U \exp \left(-c_{3} A^{3} u\right) \ll M U s^{-c_{3} A^{3}} \ll M U H^{-1},
$$

provided that $A^{3} \geq 33 /\left(2 c_{3}\right)$. With this amendment Saltykov would have obtained Walfisz' estimate (1) (see Lemma 2.8 of [7]) in a simpler way (and before Walfisz), avoiding the long and technical proof of Hilfssatz 4.4.7 in [11].

However, it seems very unlikely that Saltykov's method could, after being further amended, yield a better result than (1), as long as (26) is not proved for values of $\gamma_{1}$ and $\gamma_{2}$ with $\gamma<2 / 3$ : after all, the crucial ingredient in both arguments (Walfisz' and Saltykov's) is the same theorem of Korobov.

\section{REFERENCES}

[1] U. Balakrishnan and Y.-F.S. Pétermann, The Dirichlet series of $\zeta(s) \zeta^{\alpha}(s+1) f(s+1)$ : On an error term associated with its coefficients, Acta Arith. 75 (1996), 39-69.

[2] A. Ivić, The Riemann zeta-function, John Wiley and Sons 1985.

[3] E. Grosswald. The average order of an arithmetical function, Duke Math. J. 23 (1956), 41-44.

[4] A.A. Karatsuba, Estimates for trigonometric sums by Vinogradov's method, and some applications, Proc. Steklov Inst. Math. (A.M.S English translation, 1973) 112 (1971), 251-265.

[5] M.N. Korobov, Estimates of trigonometrical sums and their applications (in Russian), Uspekhi Mat. Nauk. 13 (4) (1958), 185-192.

[6] Y.-F.S. Pétermann and Jie Wu, On the sum of exponential divisors of an integer, Acta Math. Hungar. 77 (1997), 159-175.

[7] A.I. Saltykov, On Euler's function" (in Russian), Vestnik Moskovskogo Universiteta, Seriya I: Matematika, Mekhanika, no vol. number, fasc. number 6 (1960), 34-50.

[8] G. Tenenbaum, Introduction d̀ la théorie analytique et probabiliste des nombres. Institut Elie Cartan 131990.

[9] E.C. Titchmarsh, The theory of the Riemann zeta-function, Oxford, Clarendon Press 1951; second edition revised by D.R. Heath-Brown, ibid 1986.

[10] A. Walfisz, Über die Wirksamkeit einiger Abschätzungen trigonometrischer Summen, Acta Arith. 4 (1958), 108-180.

[11] A. Walfisz, Weylsche Exponentialsummen in der neueren Zahlentheorie. VEB Deutscher Verlag der Wissenschaften, Berlin 1963.

Y.-F. S. PÉTERMANN

Section de Mathématiques

Université de Genève

2-4, rue du Lièvre, C.P. 240

1211 Genève 24 SUISSE

E-mail : petermaneibm.unige.ch 